Bryn Mawr College

Scholarship, Research, and Creative Work at Bryn Mawr

College

1999

\title{
The Effect of Laser Bandwidth on the Signal Detected in Two-Color, Resonant Four-Wave Mixing Spectroscopy
}

F. Di Teodoro

Elizabeth McCormack

Bryn Mawr College, emccorma@brynmawr.edu

Let us know how access to this document benefits you.

Follow this and additional works at: http://repository.brynmawr.edu/physics_pubs

Part of the Physics Commons

\section{Custom Citation}

F. Di Teodoro and E.F. McCormack, J. Chem. Phys. 110, 8369 (1999).

This paper is posted at Scholarship, Research, and Creative Work at Bryn Mawr College. http://repository.brynmawr.edu/physics_pubs/2 


\title{
The effect of laser bandwidth on the signal detected in two-color, resonant four-wave mixing spectroscopy
}

\author{
F. Di Teodoro and E. F. McCormack \\ Bryn Mawr College, Bryn Mawr, Pennsylvania 19010
}

(Received 4 November 1998; accepted 8 February 1999)

\begin{abstract}
The effect of laser line shape and bandwidth on the signal detected in two-color, resonant four-wave mixing (TC-RFWM) spectroscopy is determined by means of an ab initio calculation of the third-order polarization based on diagrammatic perturbation theory. Modifications to the approach previously used for the case of $\delta$-function laser line shapes are made by introducing a different treatment of the rotating wave approximation and phase-matching conditions. A three-level excitation scheme for double-resonance spectroscopy of bound and quasibound states is analyzed. In the case of Lorentzian laser line shapes, analytic expressions for the signal line profile are obtained for each excitation scheme. Analytic approximations of the signal line profile are also obtained in the case of Gaussian laser line shapes. (c) 1999 American Institute of Physics.
\end{abstract}

[S0021-9606(99)01917-0]

\section{INTRODUCTION}

As reported by several informative review papers, ${ }^{1-5}$ resonant four-wave mixing (RFWM) is a nonlinear optical technique that, in recent years, has been successfully applied to a wide variety of investigations in atomic and molecular physics including combustion and plasma diagnostic and high-resolution, gas-phase spectroscopy. Four-wave mixing spectroscopy is based upon the interaction of three laser beams in a medium of interest to produce a nonlinear polarization via the third-order term of the electric susceptibility $\left(\chi^{(3)}\right) .^{6}$ The induced polarization is the source of a fourth, coherent light beam that is radiated from the medium and detected as the signal. The spectroscopic capabilities of the process rely in the dramatic signal enhancement occurring whenever the frequency of the incident laser beams is resonant with a transition in the medium; spectra can be obtained, therefore, by frequency-scanning the input laser beams over selected resonances. When two optical fields have frequencies resonant with two different transitions the resulting four-wave mixing process is called two-colorRFWM (TC-RFWM). TC-RFWM can offer distinct advantages over linear spectroscopic techniques for studying structure and dynamics of atomic and molecular systems. Indeed, since the signal generation is based solely upon absorption, TC-RFWM can detect any excited state regardless of its decay mode (pre- or photodissociation, autoionization, fluorescence, and internal energy-conversion). Further, the highly directional and coherent nature of the signal permits efficient rejection of background source emission and scattered laser light leading to favorable signal to noise ratios. Also, high spectral, spatial, and temporal sensitivity can be obtained for a range of pressures and number densities and low detection limits in the range of $10^{9}-10^{12}$ molecules $\mathrm{cm}^{-3}$ per quantum state have been observed. ${ }^{7}$ A distinctive characteristic of TCRFWM is that, being a doubly-resonant spectroscopic scheme, it is particularly amenable to state selection. ${ }^{8}$ These attributes have led to successful application of TC-RFWM to the study of numerous stable and transient species. ${ }^{8-22}$

Diagrammatic perturbation theory (DPT) has proven to be a very effective tool in analyzing the spectra obtained in gas-phase TC-RFWM. Analytic expressions for the signal line profile have been reported for a variety of TC-RFWM schemes used in double-resonance spectroscopy of bound ${ }^{23}$ and quasibound ${ }^{24}$ molecular states. However, the application of DPT has been carried out, so far, by assuming ideal experimental conditions. ${ }^{23}$ Saturation effects arising from the utilization of high-intensity lasers are, for example, not accounted for by this theory. Neither are nonlinear phenomena such as the formation of nonresonant, laser-induced thermal, ${ }^{25}$ acoustic $^{13}$ or electrostrictive ${ }^{26}$ gratings consisting of modulations in the real part of the refractive index that occur, in relatively high-density media, as a result of the optical interference of intense laser beams. ${ }^{27}$ In addition, when the Doppler effect is taken into account, the singlemolecule contribution to $\chi^{(3)}$, explicitly calculated by DPT, must be integrated over the velocity distribution of the absorbing molecules and, as a consequence, the analytic character of the signal line profile is lost. ${ }^{23}$ Finally, no attempt has been made to include in the DPT model the effect of finite laser bandwidths.

Many of these theoretical limitations, however, do not represent a real problem for the interpretation of the observed spectra provided that the experimental conditions are carefully selected and monitored. Indeed, saturation can be avoided as long as the signal is found to be proportional to the intensity of the probe laser and to the squared intensity of the grating laser ${ }^{16}$ and significant signal to noise ratios have been achieved in unsaturated conditions. ${ }^{10}{ }^{\text {Further, perform- }}$ ing TC-RFWM in low-pressure, collision-free environments such as supersonic molecular beams leads to a strong reduction of the excited-state collisional relaxation rate so that the rate of single-molecule decay processes (such as autoionization, dissociation, isomerization) can be obtained from the 
observed spectral lines without the need of any modification to the DPT treatment. In addition, Butenhoff et al. ${ }^{10}$ have shown that the nonresonant background signal originating from thermal and acoustic gratings and other pressure effects is practically eliminated as a consequence of the low number density characterizing the molecular beam. Thermal and electrostrictive gratings can be avoided by using input laser beams set up to have polarizations orthogonal to one another. ${ }^{28}$ Also, TC-RFWM geometries can be tailored to drastically reduce the first-order Doppler effect. ${ }^{29}$

The effect of laser line shape and bandwidth on the signal profile, however, cannot be easily experimentally circumvented as gas-phase, TC-RFWM spectroscopy is usually performed by utilizing laser-pumped, pulsed dye lasers. These laser sources exhibit a relatively broad line shape determined by numerous factors such as pulse-to-pulse variations in the pumping-laser intensity, thermooptical effects on the dye cell exposed to the pumping light, cavity longitudinal mode competition, mechanical instabilities of the cavity, and (when used) nonlinear frequency doubling of the final laser output in birefringent crystals. These phenomena cause frequency chirp and stochastic fluctuations of the phase and amplitude of the optical field resulting in typical bandwidths of $0.1-0.01 \mathrm{~cm}^{-1},{ }^{8-16,18-20}$ a value comparable to molecular transition widths in many situations of interest. Also, depending on the characteristics of the pumping laser and the active medium as well as on the optical resonator design, the dye-laser line shape may either appear as a sharp peak centered at the selected frequency or, in the opposite extreme, show a significant, irregularly structured background produced by amplified fluorescence. Suitable mathematical models for the different line shapes are either Lorentzian or Gaussian functions; the choice between the two functions being dictated by the statistical properties of the lightgenerating process. A Lorentzian line shape is appropriate when all fluctuations and relaxation processes are Markovian in nature thereby having exponential temporal autocorrelation and obeying Langevin dynamical equations, while a Gaussian line shape adequately describes the non-Markovian case (frequently termed also "nonimpact limit" 30 ) that characterizes short-pulse lasers. From a spectroscopic point of view, the distinction between the two line-shape models is very relevant and may lead to different predictions for the signal spectral profile. In the case of Lorentzian line shapes, resonant photons may be absorbed by the medium from the wings of the laser line shape resulting in non-negligible incoherent contributions to the excitation of a given state even for large center-line detunings from the corresponding transition frequency. ${ }^{31}$ Conversely, a laser with a line shape falling off faster than a Lorentzian (for example, a Gaussian line shape) is expected to appear substantially monochromatic to the medium while tuned out of resonance. It is therefore important to treat the two cases separately, even though, in practice, determining which case applies to a specific laser can be a difficult task, especially for pulsed lasers.

A time-domain analysis of the effect of laser line shape and bandwidth on the RFWM signal profile has been carried out by Smith and co-workers ${ }^{32,33}$ in the specific case of degenerate four-wave mixing, namely, an implementation of
RFWM in which all optical fields have the same frequency. This analysis consisted of integrating the rate equations for the material density matrix with the assumption that the input laser fields are incoherent, uncorrelated, and statistically independent (chaotic) Markovian fields characterized by a Lorentzian line shape with a bandwidth much larger than the characteristic widths of all processes governing the dynamics of the material system. ${ }^{34}$ Note that in the TC-RFWM experiments considered here, however, spectra are obtained by frequency scanning one of the input laser beams (the probe, as explained below) over selected resonant features, while the analysis in Refs. 32,33 is concerned with the line shape of the four-wave mixing signal radiating from a medium excited by a fixed frequency, broadband laser. In addition, we present a markedly distinct computational approach to the problem. The contribution of the laser bandwidth to the TCRFWM signal profile is determined by a full frequencydomain calculation based on DPT and formulated as an extension of the theory presented in Refs. 23,24. Both Lorentzian and Gaussian line shapes are discussed with no assumption on the relative laser bandwidth and transition widths and the theoretical treatment addresses three-level systems featuring either discrete or quasibound states.

The remainder of this paper is organized as follows. In Sec. II we derive the intensity of the TC-RFWM signal emerging from a three-level system excited by finitebandwidth input lasers with a generic line shape. In Sec. II A 1 the general expression of the third-order polarization is recalled; in Sec. II A 2 the geometric aspects of the fourwave mixing process involving finite-bandwidth lasers are discussed; and in Sec. II A 3 the application of the rotating wave approximation, which extracts the resonant part of the electric susceptibility, is carried out for an excitation scheme featuring three discrete states. Details of the derivation of the signal beam intensity and direction are presented in Appendix A, while, in Appendix B, we show that the signal is affected only by the line shape of the probe laser which is frequency-scanned over a given transition. In Sec. II B the calculations of Sec. II A are adapted to a three-level system featuring a quasibound state. In Sec. III explicit expressions for the signal profile are obtained by modeling the probe laser line shape as a Lorentzian and a Gaussian function. Computational details are shown in Appendix C. Finally, in Sec. IV we summarize our results.

\section{THEORY}

\section{A. Discrete states}

The excitation scheme that will be considered in this section is depicted in Fig. 1(a). The medium of interest exhibits three relevant discrete energy levels corresponding to bound states of atoms or molecules. The three levels are arranged in a "cascade" configuration, which is particularly suitable for spectroscopy of highly-excited states such as Rydberg states. Only straightforward computational modifications, however, are necessary to adapt the theoretical treatment developed below to different excitation schemes, a list of which is presented in Ref. 23. 


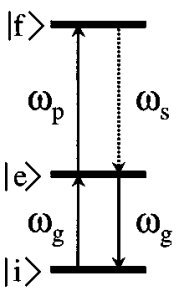

( a )

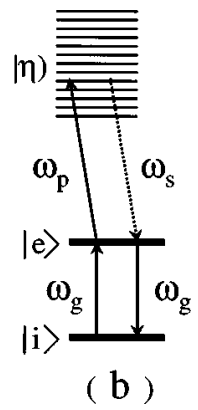

(b)
FIG. 1. Excitation schemes discussed in the text; (a) a three-level system with only discrete states and (b) a three-level system in which the highest level is a quasibound state.

\section{Polarization}

The optical field at a given point $\mathbf{r}$ and time $t, \mathbf{E}(\mathbf{r}, t)$, associated with the laser beam detected as the signal in a TC-RFWM process is, neglecting dispersion, given by the wave equation ${ }^{35}$

$$
\nabla^{2} \mathbf{E}(\mathbf{r}, t)=\frac{4 \pi}{c^{2}} \frac{\partial^{2} \mathbf{P}^{(3)}(\mathbf{r}, t)}{\partial t^{2}}+\frac{1}{c^{2}} \frac{\partial^{2} \mathbf{E}(\mathbf{r}, t)}{\partial t^{2}},
$$

where $\mathbf{P}^{(3)}(\mathbf{r}, t)$ is the third-order polarization induced in the medium by the incident fields. The polarization is nonzero only for $|\mathbf{r}|<\mathcal{V}$, where $\mathcal{V}$ is the volume of the region occupied by the medium. Assuming that all incident fields are linearly polarized along a common direction in the laboratory reference frame and that the particles forming the absorbing medium have negligible intrinsic dipoles, the only significant component of $\mathbf{P}^{(3)}(\mathbf{r}, t)$ is the one parallel to the polarization direction of the incident fields and is given by

$$
\begin{aligned}
P^{(3)}(\mathbf{r}, t)= & \frac{1}{(2 \pi)^{12}} \int d \mathbf{k}_{1} \int d \mathbf{k}_{2} \int d \mathbf{k}_{3} \int_{-\infty}^{\infty} d \omega_{3} \int_{-\infty}^{\infty} d \omega_{2} \\
& \times \int_{-\infty}^{\infty} d \omega_{1} \chi^{(3)}\left(\omega_{3}, \omega_{2}, \omega_{1}\right) \mathfrak{E}\left(\mathbf{k}_{3}, \omega_{3}\right) \\
& \times \mathfrak{E}\left(\mathbf{k}_{2}, \omega_{2}\right) \mathfrak{E}\left(\mathbf{k}_{1}, \omega_{1}\right) \exp \left(-i \omega_{s} t+i \mathbf{k}_{s} \cdot \mathbf{r}\right) .
\end{aligned}
$$

Here, the numerical indices identify the time ordering so that $\mathfrak{E}\left(\mathbf{k}_{m}, \omega_{m}\right)$ is the space-time Fourier transform of the $m$ th optical field interacting with the medium and the abbreviations $\omega_{s}=\omega_{3}+\omega_{2}+\omega_{1}$ and $\mathbf{k}_{s}=\mathbf{k}_{3}+\mathbf{k}_{2}+\mathbf{k}_{1}$ have been used.

The expression of the third-order, scalar electric susceptibility $\chi^{(3)}\left(\omega_{3}, \omega_{2}, \omega_{1}\right)$ is obtained by adapting the general expression of Ref. 36 to the excitation scheme of Fig. 1(a). By assuming that the thermal equilibrium population of all matter states is negligible except for the lowest state $|i\rangle$, we obtain

$$
\begin{aligned}
\chi^{(3)}\left(\omega_{3}, \omega_{2}, \omega_{1}\right)= & -\frac{\rho_{i i}^{0} N}{6 \hbar^{3}} \sum_{\text {perm }} \sum_{a b c=i, e, f}\langle i|\mu| a\rangle\langle a|\mu| b\rangle\langle b|\mu| c\rangle\langle c|\mu| i\rangle\left[\mathcal{G}^{c b}\left(\omega_{3}+\omega_{2}+\omega_{1}\right) \mathcal{G}^{c a}\left(\omega_{2}+\omega_{1}\right) \mathcal{G}^{c i}\left(\omega_{1}\right)\right. \\
& +\mathcal{G}^{c b}\left(\omega_{3}+\omega_{2}+\omega_{1}\right) \mathcal{G}^{c a}\left(\omega_{2}+\omega_{1}\right) \mathcal{G}^{i a}\left(\omega_{1}\right)+\mathcal{G}^{c b}\left(\omega_{3}+\omega_{2}+\omega_{1}\right) \mathcal{G}^{i b}\left(\omega_{2}+\omega_{1}\right) \mathcal{G}^{i a}\left(\omega_{1}\right)+\mathcal{G}^{a i}\left(\omega_{3}+\omega_{2}\right. \\
& \left.+\omega_{1}\right) \mathcal{G}^{b i}\left(\omega_{2}+\omega_{1}\right) \mathcal{G}^{c i}\left(\omega_{1}\right)-\mathcal{G}^{c b}\left(\omega_{3}+\omega_{2}+\omega_{1}\right) \mathcal{G}^{d b}\left(\omega_{2}+\omega_{1}\right) \mathcal{G}^{i a}\left(\omega_{1}\right)-\mathcal{G}^{b a}\left(\omega_{3}+\omega_{2}+\omega_{1}\right) \mathcal{G}^{c a}\left(\omega_{2}\right. \\
& \left.\left.+\omega_{1}\right) \mathcal{G}^{c i}\left(\omega_{1}\right)-\mathcal{G}^{b a}\left(\omega_{3}+\omega_{2}+\omega_{1}\right) \mathcal{G}^{b i}\left(\omega_{2}+\omega_{1}\right) \mathcal{G}^{c i}\left(\omega_{1}\right)-\mathcal{G}^{i c}\left(\omega_{3}+\omega_{2}+\omega_{1}\right) \mathcal{G}^{i b}\left(\omega_{2}+\omega_{1}\right) \mathcal{G}^{i a}\left(\omega_{1}\right)\right]
\end{aligned}
$$

where $\rho_{i i}^{0}$ is the diagonal element of the material density matrix corresponding to the thermal-equilibrium population of the state $|i\rangle$ and $N$ is the number density of the medium. The first summation accounts for the six possible permutations of $\omega_{1}, \omega_{2}$, and $\omega_{3}$ while $a, b$, and $c$ in the second summation are matter state indices which may take as values " $i$, ," " $e$," or " $f$. " In addition, $\mu$ is the component of the single-particle dipole operator along the common polarization direction of the incident fields and, in the square brackets, eight triple products of Green propagators account for the eight possible pathways of the three-step excitation of the medium in the four-wave mixing process. ${ }^{36}$ Each of these Green propagators is given by $\mathcal{G}^{a b}(\omega)=\left(\omega-\omega_{a b}\right.$ $\left.+i \Gamma_{a b}\right)^{-1}$, where $\omega=\omega_{1}, \omega_{2}+\omega_{1}$, or $\omega_{3}+\omega_{2}+\omega_{1}$; $\hbar\left|\omega_{a b}\right|$ is the energy gap between states $|a\rangle$ and $|b\rangle\left(\omega_{a b}\right.$ $\left.=-\omega_{b a}\right)$; and, $\Gamma_{a b}$ is the average decay rate of states $|a\rangle$ and $|b\rangle .^{23}$

\section{Incident fields}

The TC-RFWM schemes are often interpreted in terms of the formation of and the scattering from laser-induced gratings. The interference of two nearly copropagating pulsed laser beams overlapped at a small crossing angle in a medium and resonant with an energy transition produces a modulation of the optical properties of the medium that constitutes a diffraction grating. Thus, a third beam, delayed in time with respect to the previous ones, is scattered off such a laser-induced grating and the scattered beam is detected as the four-wave mixing signal. According to the nomenclature traditionally used in this description, the first two beams are labeled as grating beams and the third beam is labeled as the probe beam. The grating beams, with center-line frequency $\omega_{g}$, are tuned upon the transition $|i\rangle \rightarrow|e\rangle$ while the probebeam, whose center-line frequency is $\omega_{p}$, is delayed in time 
with respect to the grating beams and frequency-tuned to scan over the transition $|e\rangle \rightarrow|f\rangle$.

The incident fields considered in this paper are adequately described as nonmonochromatic, unidirectional wave packets. Hence, assuming the same linear polarization for all laser beams and neglecting dispersion, the Fouriertransformed optical fields $\mathfrak{E}\left(\mathbf{k}_{3}, \omega_{3}\right), \mathfrak{E}\left(\mathbf{k}_{2}, \omega_{2}\right)$, and $\mathfrak{E}\left(\mathbf{k}_{1}, \omega_{1}\right)$ have the form

$$
\begin{aligned}
& \mathfrak{E}\left(\mathbf{k}_{1}, \omega_{1}\right)=\mathcal{E}^{g} \frac{2 \delta\left(k_{1}-\omega_{1} / c\right)}{k_{1}^{2}} \delta\left(\hat{\mathbf{k}}_{1}-\hat{\mathbf{k}}_{g 1}\right) g\left(\omega_{1}-\omega_{g}, \Delta_{g}\right)+\mathcal{E}^{g *} \frac{2 \delta\left(k_{1}-\omega_{1} / c\right)}{k_{1}^{2}} \delta\left(\hat{\mathbf{k}}_{1}+\hat{\mathbf{k}}_{g 1}\right) g\left(\omega_{1}+\omega_{g}, \Delta_{g}\right), \\
& \mathfrak{E}\left(\mathbf{k}_{2}, \omega_{2}\right)=\mathcal{E}^{g} \frac{2 \delta\left(k_{2}-\omega_{2} / c\right)}{k_{2}^{2}} \delta\left(\hat{\mathbf{k}}_{2}-\hat{\mathbf{k}}_{g 2}\right) g\left(\omega_{2}-\omega_{g}, \Delta_{g}\right)+\mathcal{E}^{g *} \frac{2 \delta\left(k_{2}-\omega_{2} / c\right)}{k_{2}^{2}} \delta\left(\hat{\mathbf{k}}_{2}+\hat{\mathbf{k}}_{g 2}\right) g\left(\omega_{2}+\omega_{g}, \Delta_{g}\right), \\
& \mathfrak{E}\left(\mathbf{k}_{3}, \omega_{3}\right)=\mathcal{E}^{p} \frac{2 \delta\left(k_{3}-\omega_{3} / c\right)}{k_{3}^{2}} \delta\left(\hat{\mathbf{k}}_{3}-\hat{\mathbf{k}}_{p}\right) p\left(\omega_{3}-\omega_{p}, \Delta_{p}\right)+\mathcal{E}^{p *} \frac{2 \delta\left(k_{3}-\omega_{3} / c\right)}{k_{3}^{2}} \delta\left(\hat{\mathbf{k}}_{3}+\hat{\mathbf{k}}_{p}\right) p\left(\omega_{3}+\omega_{p}, \Delta_{p}\right),
\end{aligned}
$$

where $\mathcal{E}^{g}$ and $\mathcal{E}^{p}$ are the complex amplitudes of the grating and probe beams, respectively, and the wave vectors $\mathbf{k}_{m}(m$ $=1,2,3)$ have been written as $\mathbf{k}_{m}=k_{m} \hat{\mathbf{k}}_{m}$, where $k_{m}$ is the magnitude of the wave vector and $\hat{\mathbf{k}}_{m}$ is a unit vector in momentum space oriented along the direction of $\mathbf{k}_{m}$. Further, $\hat{\mathbf{k}}_{g 1}, \hat{\mathbf{k}}_{g 2}$ are unit vectors parallel to the propagation direction of the grating beams and $\hat{\mathbf{k}}_{p}$ is a unit vector parallel to the propagation direction of the probe beam. The functions $g$ and $p$ in Eq. (4) represent the spectral profile of the grating and probe beams, respectively. The functions $g\left(\omega_{1}\right.$ $\left.\pm \omega_{g}, \Delta_{g}\right)$ and $p\left(\omega_{3} \pm \omega_{p}, \Delta_{p}\right)$ are centered at $\mp \omega_{g}$ and $\mp \omega_{p}$, respectively, with characteristic bandwidths $\Delta_{g}$ and $\Delta_{p}$. The integration over $\mathbf{k}_{1}, \mathbf{k}_{2}$, and $\mathbf{k}_{3}$ in Eq. (2) may be immediately performed by introducing spherical coordinates in momentum space

$$
\int d \mathbf{k}_{m}=\int_{0}^{\infty} k_{m}^{2} d k_{m} \int d \hat{\mathbf{k}}_{m},
$$

where, again, $m=1,2,3$ and the integral over $\hat{\mathbf{k}}_{m}$ is extended to the entire solid angle in momentum space. Substituting Eq. (4) into Eq. (2) and making use of Eq. (5) yields

$$
\begin{aligned}
P^{(3)}(\mathbf{r}, t)= & \frac{1}{(2 \pi)^{12}} \int_{-\infty}^{\infty} d \omega_{3} \int_{-\infty}^{\infty} d \omega_{2} \int_{-\infty}^{\infty} d \omega_{1} \chi^{(3)}\left(\omega_{3}, \omega_{2}, \omega_{1}\right) \exp \left(-i \omega_{s} t\right)\left[\mathcal{E}^{p} p\left(\omega_{3}-\omega_{p}, \Delta_{p}\right) \exp \left(i \frac{\omega_{3}}{c} \hat{\mathbf{k}}_{p} \cdot \mathbf{r}\right)\right. \\
& \left.+\mathcal{E}^{p *} p\left(\omega_{3}+\omega_{p}, \Delta_{p}\right) \exp \left(-i \frac{\omega_{3}}{c} \hat{\mathbf{k}}_{p} \cdot \mathbf{r}\right)\right]\left[\mathcal{E}^{g} g\left(\omega_{2}-\omega_{g}, \Delta_{g}\right) \exp \left(i \frac{\omega_{2}}{c} \hat{\mathbf{k}}_{g 2} \cdot \mathbf{r}\right)+\mathcal{E}^{g *} g\left(\omega_{2}+\omega_{g}, \Delta_{g}\right)\right. \\
& \left.\times \exp \left(-i \frac{\omega_{2}}{c} \hat{\mathbf{k}}_{g 2} \cdot \mathbf{r}\right)\right]\left[\mathcal{E}^{g} g\left(\omega_{1}-\omega_{g}, \Delta_{g}\right) \exp \left(i \frac{\omega_{1}}{c} \hat{\mathbf{k}}_{g 1} \cdot \mathbf{r}\right)+\mathcal{E}^{g *} g\left(\omega_{1}+\omega_{g}, \Delta_{g}\right) \exp \left(-i \frac{\omega_{1}}{c} \hat{\mathbf{k}}_{g 1} \cdot \mathbf{r}\right)\right]
\end{aligned}
$$

As shown in Appendix A, if the input fields were perfectly monochromatic, i.e., if the functions $p$ and $g$ in Eq. (6) were replaced by $\delta$-functions centered on $\omega_{p}$ and $\omega_{g}$, respectively, the induced polarization oscillating at frequency $\omega_{p}$ could be written as a plane wave propagating along the direction illustrated in Fig. 2(a) and specified by Eq. (A10). Since the input fields have finite spectral bandwidths, however, the induced polarization resulting from Eq. (6) should be described as a nonmonochromatic wave packet. The functions $p$ and $g$ peak at $\omega_{p}$ and $\omega_{g}$ and then fall rapidly to zero outside the bandwidth range, such that the wave packet has a maximum amplitude at the angle given by Eq. (A10). Unlike the unidirectional input fields, however, it exhibits a geometrical spread around this angle. This spread is because the wave vectors embedded in each of the input-field wave packets, oriented along a well defined direction but with different magnitudes, combine vectorially in the mixing process as illustrated in Fig. 2(b). Simple geometry, however, shows that the typical angular dispersion, $\Delta \theta$, of the polarization wave packet is roughly on the order of $\left(\Delta_{g}+\Delta_{p}\right) /\left(\omega_{g}\right.$ $\left.+\omega_{p}\right)$ which gives $\Delta \theta \ll 1$ for realistic laser sources. The phase-matched TC-RFWM signal beam has the same spatial characteristics of the induced polarization (see Appendix A) and exhibits, therefore, the same angular dispersion $\Delta \theta$. The angular acceptance of the detector used to collect the fourwave mixing signal is far too large to resolve $\Delta \theta$ and, as a result, the observed signal is equivalent to that from a plane wave propagating along the direction given by the angle in 
Eq. (A10). Accordingly, we assume that the spatial characteristics of the mixing process, namely the orientation of the laser-induced grating and the direction of the light scattered off from this grating, can be adequately described, as in the case of $\delta$-function laser beams, by associating only the "center-line"' wave vectors $\left(\omega_{g} / c\right) \hat{\mathbf{k}}_{g 1,2}$ and $\left(\omega_{p} / c\right) \hat{\mathbf{k}}_{p}$ with the grating and probe beams, respectively. This allows us to rewrite Eq. (6) as

$$
\begin{aligned}
& P^{(3)}(\mathbf{r}, t)=\frac{1}{(2 \pi)^{12}} \int_{-\infty}^{\infty} d \omega_{3} \int_{-\infty}^{\infty} d \omega_{2} \int_{-\infty}^{\infty} d \omega_{1} \chi^{(3)}\left(\omega_{3}, \omega_{2}, \omega_{1}\right) \exp \left(-i \omega_{s} t\right)\left[\mathcal{E}^{p} p\left(\omega_{3}-\omega_{p}, \Delta_{p}\right) \exp \left(i \frac{\omega_{p}}{c} \hat{\mathbf{k}}_{p} \cdot \mathbf{r}\right)\right. \\
& \left.+\mathcal{E}^{p *} p\left(\omega_{3}+\omega_{p}, \Delta_{p}\right) \exp \left(-i \frac{\omega_{p}}{c} \hat{\mathbf{k}}_{p} \cdot \mathbf{r}\right)\right]\left[\mathcal{E}^{g} g\left(\omega_{2}-\omega_{g}, \Delta_{g}\right) \exp \left(i \frac{\omega_{g}}{c} \hat{\mathbf{k}}_{g 2} \cdot \mathbf{r}\right)+\mathcal{E}^{g *} g\left(\omega_{2}+\omega_{g}, \Delta_{g}\right)\right. \\
& \left.\times \exp \left(-i \frac{\omega_{g}}{c} \hat{\mathbf{k}}_{g 2} \cdot \mathbf{r}\right)\right]\left[\mathcal{E}^{g} g\left(\omega_{1}-\omega_{g}, \Delta_{g}\right) \exp \left(i \frac{\omega_{g}}{c} \hat{\mathbf{k}}_{g 1} \cdot \mathbf{r}\right)+\mathcal{E}^{g *} g\left(\omega_{1}-\omega_{g}, \Delta_{g}\right) \exp \left(-i \frac{\omega_{g}}{c} \hat{\mathbf{k}}_{g 1} \cdot \mathbf{r}\right)\right] \text {. }
\end{aligned}
$$

\section{Rotating-wave approximation}

The evaluation of the triple frequency-integral in Eq. (7) can be performed by using the rotating wave approximation (RWA) to extract the resonant susceptibility terms from Eq. (3). The application of the RWA can be simplified by using the double-sided (DS) Feynman diagram formalism. ${ }^{36}$ Only the two diagrams depicted in Fig. 3 are needed to properly describe the excitation scheme of Fig. 1(a) and their physical meaning can be described as follows. Initially, negligible population characterizes all states other than $|i\rangle$. The thermal-equilibrium material density operator is $\rho_{i i}^{0}|i\rangle\langle i|$, such that the first interaction is the absorption $|i\rangle \rightarrow|e\rangle$ stimulated by the grating beams that create the coherences $\rho_{e i}|e\rangle\langle i|$ and $\rho_{i e}|i\rangle\langle e|$. The second interaction is again the absorption $|i\rangle \rightarrow|e\rangle$ and the third interaction is the absorption $|e\rangle \rightarrow|f\rangle$. Only the two time-orderings are needed to describe the TC-RFWM scheme under examination because the probe beam, which is frequency-tuned on the transition $|e\rangle \rightarrow|f\rangle$, is temporally delayed with respect to the grating light pulses and will affect the mixing process only after the grating light pulses, which are tuned on the transition $|i\rangle$ $\rightarrow|e\rangle$, interact with the medium. In addition, even though the input lasers have finite bandwidths, the optical fields, once frequency-tuned on a specific transition of the excitation scheme of Fig. 1(a), are not spectrally broad enough to interact with the other transition as long as the inequalities

$$
\begin{aligned}
& \Delta_{g} \ll\left|\omega_{g}-\omega_{p}\right|, \\
& \Delta_{p} \ll\left|\omega_{g}-\omega_{p}\right|,
\end{aligned}
$$

hold. Thus, the two grating beams in the mixing process generate the population $\rho_{e e}|e\rangle\langle e|$ that exhibits spatial modulation perpendicular to the directions $\pm\left(\omega_{g} / c\right)\left(\hat{\mathbf{k}}_{g 1}-\hat{\mathbf{k}}_{g 2}\right)$. After the delay time, the probe beam interacts with the medium by exciting the transition $|e\rangle \rightarrow|f\rangle$ such that the coherences $\rho_{f e}|f\rangle\langle e|$ and $\rho_{e f}|e\rangle\langle f|$ are created. This implies, according to Eq. (7), that the induced polarization will consist of only two terms characterized by the spatial-phase factors $\exp \left(i \mathbf{k}_{s} \cdot \mathbf{r}\right)$ and $\exp \left(i \mathbf{k}_{s}^{\prime} \cdot \mathbf{r}\right)$, where

$$
\mathbf{k}_{s}=\left(\omega_{p} / c\right) \hat{\mathbf{k}}_{p}+\left(\omega_{g} / c\right)\left(\hat{\mathbf{k}}_{g 1}-\hat{\mathbf{k}}_{g 2}\right),
$$

$$
\mathbf{k}_{s}^{\prime}=\left(\omega_{p} / c\right) \hat{\mathbf{k}}_{p}-\left(\omega_{g} / c\right)\left(\hat{\mathbf{k}}_{g 1}-\hat{\mathbf{k}}_{g 2}\right) .
$$

As shown in Appendix A, however, the frequency phasematching condition $\left|\mathbf{k}_{s}\right| \simeq \omega_{p} / c$ uniquely determines the wave vector and only the coherence $\rho_{f e}|f\rangle\langle e|$ will contribute.

The resonant part of the third-order susceptibility, represented by the two DS Feynman diagrams depicted in Fig. 3, is given by

$$
\begin{aligned}
\chi^{(3)}\left(\omega_{3}, \omega_{2}, \omega_{1}\right) \\
=S|\langle i|\mu| e\rangle|^{2}|\langle e|\mu| f\rangle|^{2} \frac{1}{\omega_{3}-\omega_{f e}+i \Gamma_{f e}} \\
\quad \times\left(\frac{1}{\omega_{1}-\omega_{e i}+i \Gamma_{e i}}+\frac{1}{-\omega_{1}+\omega_{e i}+i \Gamma_{e i}}\right),
\end{aligned}
$$

where $S \equiv\left(\rho_{i i}^{0} N\right) /\left(6 i \hbar^{3} \Gamma_{e e}\right)$. Thus, Eq. (7) can be written as

$$
\begin{aligned}
P^{(3)}(\mathbf{r}, t) & \\
= & P \exp \left(i \mathbf{k}_{s} \cdot \mathbf{r}\right) \int_{-\infty}^{\infty} d \omega_{3} \int_{-\infty}^{\infty} d \omega_{2} \int_{-\infty}^{\infty} d \omega_{1} \exp \left(-i \omega_{s} t\right) \\
& \times\left\{\frac{p\left(\omega_{3}-\omega_{p}, \Delta_{p}\right) g\left(\omega_{2}+\omega_{g}, \Delta_{g}\right) g\left(\omega_{1}-\omega_{g}, \Delta_{g}\right)}{\left(\omega_{3}-\omega_{f e}+i \Gamma_{f e}\right)\left(\omega_{1}-\omega_{e i}+i \Gamma_{e i}\right)}\right. \\
& \left.+\frac{p\left(\omega_{3}-\omega_{p}, \Delta_{p}\right) g\left(\omega_{2}-\omega_{g}, \Delta_{g}\right) g\left(\omega_{1}+\omega_{g}, \Delta_{g}\right)}{\left(\omega_{3}-\omega_{f e}+i \Gamma_{f e}\right)\left(-\omega_{1}+\omega_{e i}+i \Gamma_{e i}\right)}\right\},
\end{aligned}
$$

where $\quad P \equiv\left[S /(2 \pi)^{12}\right] \mathcal{E}^{p}\left|\mathcal{E}^{g}\right|^{2}|\langle i|\mu| e\rangle|^{2}|\langle e|\mu| f\rangle|^{2}$. As shown in Appendix $\mathrm{B}$, the double integration over $\omega_{1}$ and $\omega_{2}$ can be carried out at this point and it does not affect the line profile of the detected signal. Next, by assuming that the signal is detected at the origin of the reference frame, i.e., $\mathbf{r}=0$, the Fourier transform of the polarization can be expressed as

$$
P^{(3)}(\mathbf{r}, \omega)=\frac{P}{\Gamma_{e i}} \exp \left(i \mathbf{k}_{s} \cdot \mathbf{r}\right) \frac{p\left(\omega-\omega_{p}, \Delta_{p}\right)}{\left(\omega-\omega_{f e}+i \Gamma_{f e}\right)},
$$


where the numerical frequency-index has been dropped for simplicity. And finally, as shown in Appendix A, the intensity $I$ of the detected signal for the excitation scheme depicted in Fig. 1(a) is given by

$$
I=\frac{|P|^{2} L^{2} \omega_{f e}^{2}}{8 \pi \Gamma_{e i}^{2} c}\left|\int_{-\infty}^{\infty} d \omega \frac{p\left(\omega-\omega_{p}, \Delta_{p}\right)}{\left(\omega-\omega_{f e}+i \Gamma_{f e}\right)}\right|^{2}
$$

where $L$ is the length of the path of the output beam through the medium.

\section{B. Quasibound resonances}

\section{Configuration-interaction theory}

Quasibound states arise from the interaction of discrete and continuum states of atoms and molecules and occur, for example, in phenomena such as autoionization and predissociation. The mixed nature of quasibound states can result in asymmetric resonant features in measurements of photoemission, photoabsorption, photodetachment, and photofragmentation due to competing processes that govern the decay dynamics of the various states. The interaction of a single, isolated discrete level with a single continuum can often be described by a configuration-interaction (CI) treatment ${ }^{37}$ and this model will be adopted here. The state $|Q B\rangle$ can undergo autoionization or predissociation by decaying into an ionization or dissociation continuum with a rate $\Gamma$ much larger than the decay rates of ordinary bound states. In the CI treatment, neither $|Q B\rangle$ nor the interacting continuum are exact eigenstates of the field-free Hamiltonian. The Hamiltonian is, instead, diagonalized by a continuous manifold of mixed states called Fano states. ${ }^{37,38}$ A Fano state is indicated hereafter by $\mid \eta)$, with the reduced-energy index $\eta$ defined as

$$
\eta=\frac{\omega_{\eta e}-\omega_{Q B}}{\Gamma / 2},
$$

where $\hbar \omega_{\eta e}$ is the energy separation between the Fano state and the intermediate state $|e\rangle$, and $\hbar \omega_{Q B}$ is the energy separation between the quasibound state $|Q B\rangle$ and $|e\rangle$ plus a small energy shift that depends on $\Gamma$ and is often negligible. The spectral density of the dipole transition probability for photoexcitation $|e\rangle \rightarrow \mid \eta)$ is then given by

$$
|\langle e|\mu| \eta)|^{2}=p_{c} \frac{(q+\eta)^{2}}{1+\eta^{2}}+p_{u}
$$

where $p_{c}$ is the probability of direct photoionization (or photodissociation) into the CI continuum, $p_{u}$ is the probability of transitions to other continua uncoupled to the quasibound state, and $q$ is the asymmetry parameter. ${ }^{38}$ The values of $p_{c}$, $q, p_{u}$, and $\Gamma$ may be considered to be nearly $\eta$-independent for a range of values about $\eta=0$ and treated as fitting parameters. $^{37}$

\section{Polarization}

The excitation scheme to be considered here is depicted in Fig. 1(b) and is characterized by the presence of the continuous manifold of Fano states $\mid \eta$ ) in place of the bound state $|f\rangle$. The expression of the third-order susceptibility is obtained by summing the contributions of all Fano states, ${ }^{24}$ i.e., integrating Eq. (9) over $\eta$ to give

$$
\begin{aligned}
\chi^{(3)}\left(\omega_{3}, \omega_{2}, \omega_{1}\right) \\
=S|\langle i|\mu| e\rangle|^{2} \int_{-\infty}^{\infty} d \eta|\langle e|\mu| \eta)|^{2}\left[\frac{1}{\omega_{3}-\omega_{\eta e}+i \Gamma / 2}\right. \\
\left.\quad \times\left(\frac{1}{\omega_{1}-\omega_{e i}+i \Gamma_{e i}}+\frac{1}{-\omega_{1}+\omega_{e i}+i \Gamma_{e i}}\right)\right],
\end{aligned}
$$

where the approximation $\Gamma_{\eta e} \simeq \Gamma / 2$ has been used, since $\Gamma$, the decay rate of each Fano state $\mid \eta$ ), is taken to be much larger than the decay rate of the intermediate state $|e\rangle$. In light of Eq. (15), Eq. (11) becomes

$$
\begin{aligned}
P^{(3)}(\mathbf{r}, \omega)= & \frac{S \mathcal{E}^{p}\left|\mathcal{E}^{g}\right|{ }^{2}|\langle i|\mu| e\rangle|^{2}}{(2 \pi)^{12} \Gamma_{e i}} \exp \left(i \mathbf{k}_{s} \cdot \mathbf{r}\right) \\
& \times \int_{-\infty}^{\infty} d \eta\left[\frac{(q+\eta)^{2}}{1+\eta^{2}}+r\right] \frac{p\left(\omega-\omega_{p}, \Delta_{p}\right)}{\left(\omega-\omega_{\eta e}+i \Gamma / 2\right)},
\end{aligned}
$$

where $r=p_{u} / p_{c}$. As shown in Appendix A, the intensity of the detected TC-RFWM signal for the excitation scheme depicted in Fig. 1(b) is then given by

$$
\begin{aligned}
I= & \frac{|S|^{2}\left|\mathcal{E}^{p}\right|^{2}\left|\mathcal{E}^{g}\right|^{4}|\langle i|\mu| e\rangle|^{4}}{(2 \pi)^{12} \Gamma_{e i}^{2}} \frac{L^{2} \omega_{Q B}^{2}}{8 \pi c} \\
& \times\left|\int_{-\infty}^{\infty} d \omega \int_{-\infty}^{\infty} d \eta\left[\frac{(q+\eta)^{2}}{1+\eta^{2}}+r\right] \frac{p\left(\omega-\omega_{p}, \Delta_{p}\right)}{\left(\omega-\omega_{\eta e}+i \Gamma / 2\right)}\right|^{2} .
\end{aligned}
$$

This result is analogous to Eq. (12) that applies to the excitation scheme of Fig. 1(b) for discrete states.

\section{RESULTS AND DISCUSSION}

\section{A. Discrete states}

\section{Lorentzian line shape for the probe beam}

In the case of probe beam with a Lorentzian line shape, the explicit expression for $p\left(\omega-\omega_{p}, \Delta_{p}\right)$ is

$$
p\left(\omega-\omega_{p}, \Delta_{p}\right)=\frac{1}{2 \pi} \frac{\Delta_{p}}{\left(\omega-\omega_{p}\right)^{2}+\left(\Delta_{p} / 2\right)^{2}},
$$

where $\Delta_{p}$ is the full-width at half-maximum (FWHM). Thus, by introducing the reduced quantities

$$
\begin{aligned}
\zeta & \equiv \frac{\omega-\omega_{f e}}{\Gamma_{f e} / 2}, \\
\zeta_{p} & \equiv \frac{\omega_{p}-\omega_{f e}}{\Gamma_{f e} / 2}, \\
\beta & \equiv \frac{\Delta_{p}}{\Gamma_{f e}} .
\end{aligned}
$$

Equation (12) can be rewritten as 


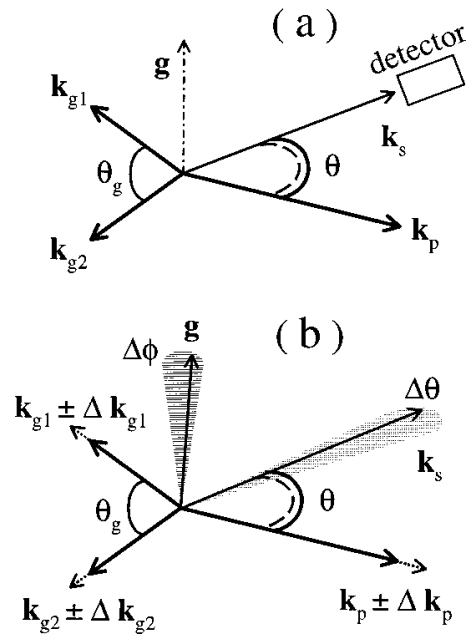

FIG. 2. Wave vector diagrams illustrating the phase-matching condition for the TC-RFWM scheme under consideration. (a) The case of $\delta$-function input laser beams, where the vector $\mathbf{g}$, called the grating vector, is given by $\mathbf{k}_{g 1}-\mathbf{k}_{g 2}$. (b) The same as (a) for the case of finite-bandwidth input laser beams. The wave vectors associated with the different plane-wave components of the input laser beams can fall at any point between the tip of the solid vector and the tip of the dashed one (the distance between the tips of solid and dashed vectors is exaggerated for illustrative purposes). The gray areas symbolize the resulting angular spreads associated with the grating vector $(\Delta \phi)$ and the propagation direction of the detected signal $(\Delta \theta)$.

$$
I=\frac{|P|^{2} L^{2} \omega_{f e}^{2}}{8 \pi c \Gamma_{e i}^{2}} \frac{4}{\pi^{2}} \frac{\Delta_{p}^{2}}{\Gamma_{f e}^{4}} \mathcal{I}\left(\zeta_{p}\right),
$$

where $\mathcal{I}\left(\zeta_{p}\right)$ is the signal line profile given by

$$
\mathcal{I}\left(\zeta_{p}\right)=\left|\int_{-\infty}^{\infty} \frac{d \zeta}{\left[\left(\zeta-\zeta_{p}\right)^{2}+\beta^{2}\right](\zeta+2 i)}\right|^{2} .
$$

The integral in Eq. (21) may be evaluated as shown in Appendix $\mathrm{C}$ and the final result is

$$
\mathcal{I}\left(\zeta_{p}\right)=\frac{\pi^{2}}{\beta^{2}} \frac{1}{\zeta_{p}^{2}+(\beta+2)^{2}} .
$$

\section{Gaussian line shape for the probe beam}

By assuming that the probe beam has a Gaussian line shape given by

$$
p\left(\omega-\omega_{p}, \Delta_{p}\right)=\frac{2 \sqrt{\ln 2}}{\Delta_{p} \sqrt{\pi}} \exp \left[-\frac{\ln 16}{\Delta_{p}^{2}}\left(\omega-\omega_{p}\right)^{2}\right],
$$

where $\Delta_{p}$ is the FWHM, and making use again of Eqs. (19), Eq. (12) can be written as

$$
I=\frac{|P|^{2} L^{2} \omega_{f e}^{2}}{8 \pi c \Gamma_{e i}^{2}} \frac{2 \sqrt{\ln 2}}{\Delta_{p} \sqrt{\pi}} \mathcal{I}\left(\zeta_{p}\right),
$$

where $\mathcal{I}\left(\zeta_{p}\right)$ is the signal line profile given by

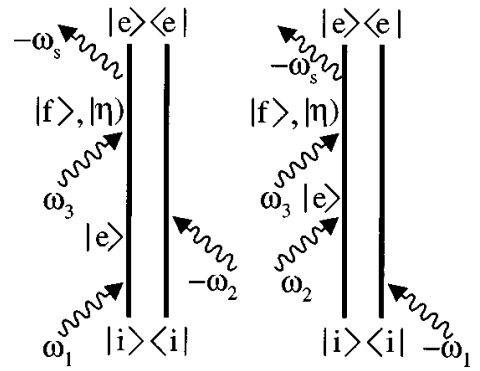

FIG. 3. Double-sided Feynman diagrams representing the resonant terms of the electric susceptibility in the TC-RFWM schemes under consideration. The final state can be either the discrete state $|f\rangle$ or the Fano state $\mid \eta)$. In the latter case, the effective susceptibility is obtained by replicating the diagrams for every $\eta$.

$$
\begin{aligned}
\mathcal{I}\left(\zeta_{p}\right)= & \left\{\int_{-\infty}^{\infty} d \zeta \exp \left[-\frac{\ln 2}{\beta^{2}}\left(\zeta-\zeta_{p}\right)^{2}\right] \frac{\zeta}{\zeta^{2}+4}\right\}^{2} \\
& +4\left\{\int_{-\infty}^{\infty} d \zeta \exp \left[-\frac{\ln 2}{\beta^{2}}\left(\zeta-\zeta_{p}\right)^{2}\right] \frac{1}{\zeta^{2}+4}\right\}^{2} .
\end{aligned}
$$

While the integrals in Eq. (25) cannot be evaluated analytically, the exponentials in Eq. (25) can be Taylor-expanded around the center-line frequency with the integration interval set to coincide with the bandwidth of the probe laser and analytic results can be obtained for every order of expansion. This procedure will provide fitting functions to analyze observed spectra. The approximation is expected to be adequate as long as $\Delta_{p} / \Gamma_{f e}<1$, i.e., when the probe laser bandwidth, albeit non-negligible, is significantly smaller than the average width of the excited states $|e\rangle$ and $|f\rangle$. Note that the lowest-order term of the Taylor expansion approximates the probe laser line shape $p\left(\omega-\omega_{p}, \Delta_{p}\right)$ as a "box" function of width $\Delta_{p}$,

$$
p\left(\omega-\omega_{p}, \Delta_{p}\right)=\left\{\begin{array}{l}
\Delta_{p}^{-1} \text { for }\left|\omega-\omega_{p}\right| \leqslant \Delta_{p} / 2 \\
0 \text { for }\left|\omega-\omega_{p}\right|>\Delta_{p} / 2
\end{array} .\right.
$$

Substituting Eq. (26) into Eq. (12) and making use of Eqs. (19) yields

$$
I=\frac{|P|^{2} L^{2} \omega_{f e}^{2}}{8 \pi c \Gamma_{e i}^{2}} \frac{1}{\Delta_{p}^{2}} \mathcal{I}\left(\zeta_{p}\right),
$$

where $\mathcal{I}\left(\zeta_{p}\right)$ is the signal line profile given by

$$
\mathcal{I}\left(\zeta_{p}\right)=\left|\int_{\zeta_{p}-\beta}^{\zeta_{p}+\beta} \frac{1}{\zeta+2 i} d \zeta\right|^{2}
$$

which, according to Eqs. (C4), (C5), and (C6) of Appendix $\mathrm{C}$, can be written as

$$
\mathcal{I}\left(\zeta_{p}\right)=\operatorname{arctanh}^{2} \frac{2 \beta \zeta_{p}}{4+\zeta_{p}^{2}+\beta^{2}}+\arctan ^{2} \frac{4 \beta}{4+\zeta_{p}^{2}-\beta^{2}} .
$$

\section{Discussion}

In Figs. 4(a) and 4(b), the normalized signal line profiles $\mathcal{I}\left(\zeta_{p}\right)$ given by Eqs. (22) and (29), respectively, are plotted 

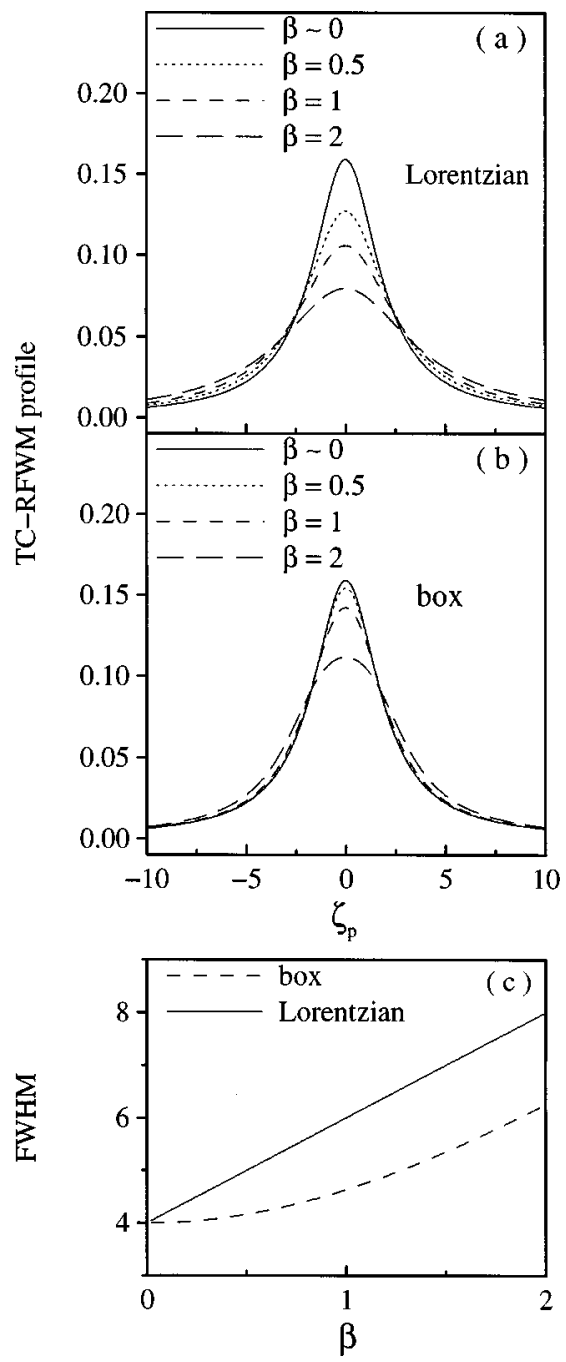

FIG. 4. TC-RFWM line profiles obtained from (a) Eq. (22) and (b) Eq. (29), for different values of $\beta$. (c) Plot of the full-width at half-maximum (FWHM) of the profiles given by Eq. (29) (dashed line) and Eq. (22) (solid line) as a function of $\beta$.

in units of $\Gamma_{f e} / 2$ for different values of the parameter $\beta$. The main aspect of the graphs is that the larger the contribution of the far wings of the probe laser line shape, the higher the sensitivity of the signal profile to the probe laser bandwidth. In order to quantitatively show this aspect, the FWHM of the signal profile obtained in the case of a Lorentzian, Eq. (22), and a boxlike, Eq. (29), probe laser line shape are plotted vs $\beta$ in Fig. 4(c). By rewriting Eq. (22) in unreduced variables, we obtain

$$
\mathcal{I}\left(\omega_{p}\right)=\frac{1}{\left(\Gamma_{f e} / 2\right)^{2}} \frac{1}{\left(\omega_{p}-\omega_{f e}\right)^{2}+\left[\left(\Delta_{p} / 2\right)+\Gamma_{f e}\right]^{2}} .
$$

From Eq. (30), it is seen that, in the case of a Lorentzian probe laser line shape, the signal profile is also a Lorentzian with a FWHM given by $\Delta_{p}+2 \Gamma_{f e}$. If the decay rate $\Gamma_{f}$ of the state $|f\rangle$ is much larger than the decay rate of $|e\rangle$, then $\Gamma_{f e} \simeq \Gamma_{f} / 2$ and the FWHM of the signal profile will be $\Delta_{p}$ $+\gamma_{f}$, i.e., the sum of the probe-laser bandwidth and the excited state width.

\section{B. Quasibound resonances}

\section{Lorentzian line shape for the probe beam}

By letting $p\left(\omega-\omega_{p}, \Delta_{p}\right)$ be a Lorentzian function given by Eq. (18) and introducing the reduced variables

$$
\begin{aligned}
& \xi \equiv \frac{\omega-\omega_{Q B}}{\Gamma / 2}, \\
& \xi_{p} \equiv \frac{\omega_{p}-\omega_{Q B}}{\Gamma / 2}, \\
& \sigma \equiv \frac{\Delta_{p}}{\Gamma},
\end{aligned}
$$

which corresponds to the TC-RFWM line profile obtained when assuming monochromatic $\delta$-like lasers. ${ }^{18}$ would give rise to a signal profile described by a Voigt profile thereby allowing for the extraction of the transition file. The a a profile provided by Eq. (29) yields, however, a valuable and simple method to obtain a direct interpretation of the trends. To test the reliability of this approximation, the signal profile arising from Eq. (29) has been normalized and plotted in Fig. different values of the parameter $\beta$, together with the excellent agreement up to $\beta \approx 1$. Both the line profile in Eq. line profile given by Eq. (26) converge, as one limit where $\beta \rightarrow 0$, where the box function in treated in this limit as a delta function.

Eq. (17) becomes

$$
I=\frac{|S|^{2}\left|\mathcal{E}^{p}\right|^{2}\left|\mathcal{E}^{g}\right|^{4}|\langle i|\mu| e\rangle|^{4}}{(2 \pi)^{12} \Gamma_{e i}^{2}} \frac{L^{2} \omega_{Q B}^{2}}{8 \pi c} \frac{4}{\pi^{2}} \frac{\Delta_{p}^{2}}{\Gamma^{4}} \mathcal{I}\left(\xi_{p}\right),
$$

where the signal line profile $\mathcal{I}\left(\xi_{p}\right)$ is given by 


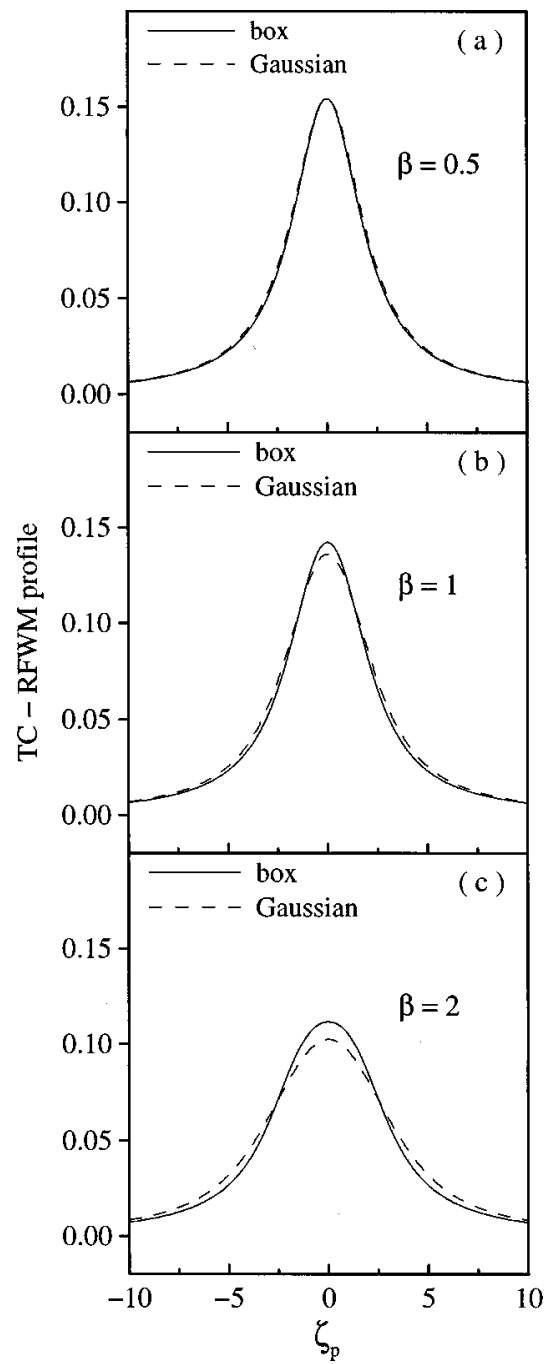

FIG. 5. Comparison of the profiles given by Eqs. (29) (solid line) and (25) (dashed line) for (a) $\beta=0.5$, (b) $\beta=1$, and (c) $\beta=2$.

$$
\begin{aligned}
\mathcal{I}\left(\xi_{p}\right)= & \mid \int_{-\infty}^{\infty} d \xi \frac{1}{\left(\xi-\xi_{p}\right)^{2}+\sigma^{2}} \int_{-\infty}^{\infty} \\
& \times\left. d \eta\left[\frac{(q+\eta)^{2}}{1+\eta^{2}}+r\right] \frac{1}{(\xi-\eta+i)}\right|^{2} .
\end{aligned}
$$

The evaluation of the integrals in Eq. (33) may be carried out as shown in Eq. (C3) of Appendix C to obtain

$$
\mathcal{I}\left(\xi_{p}\right)=\frac{\pi^{2}}{\sigma^{2}} \frac{\left[q^{2}+r+(1+r)(1+\sigma)\right]^{2}+\left[2 q+(1+r) \xi_{p}\right]^{2}}{\xi_{p}^{2}+(\sigma+2)^{2}} .
$$

\section{Gaussian line shape for the probe beam}

If $p\left(\omega-\omega_{p}, \Delta_{p}\right)$ is given by the Gaussian function in Eq. (23) and by using the reduced quantities in Eqs. (32), Eq. (17) becomes

$$
I=\frac{|S|^{2}\left|\mathcal{E}^{p}\right|^{2}\left|\mathcal{E}^{g}\right|^{4}|\langle i|\mu| e\rangle|^{4}}{(2 \pi)^{12} \Gamma_{e i}^{2}} \frac{L^{2} \omega_{Q B}^{2}}{8 \pi c} \frac{2 \sqrt{\ln 2}}{\Delta_{p} \sqrt{\pi}} \mathcal{I}\left(\xi_{p}\right),
$$

where

$$
\begin{aligned}
\mathcal{I}\left(\xi_{p}\right)= & \mid \int_{-\infty}^{\infty} d \xi \exp \left[-\frac{\ln 2}{\sigma^{2}}\left(\xi-\xi_{p}\right)^{2}\right] \\
& \times\left.\int_{-\infty}^{\infty} d \eta\left[\frac{(q+\eta)^{2}}{1+\eta^{2}}+r\right] \frac{1}{(\xi-\eta+i)}\right|^{2} .
\end{aligned}
$$

By evaluating the integral over $\eta$ as shown in Eq. (C2) of Appendix $\mathrm{C}$, the signal line profile may be written as

$$
\begin{aligned}
\mathcal{I}\left(\xi_{p}\right)= & \left\{\int_{-\infty}^{\infty} \exp \left[-\frac{\ln 2}{\sigma^{2}}\left(\xi-\xi_{p}\right)^{2}\right] \frac{\left(q^{2}-1\right) \xi-4 q}{\xi^{2}+4} d \xi\right\}^{2} \\
& +\left\{\int_{-\infty}^{\infty} \exp \left[-\frac{\ln 2}{\sigma^{2}}\left(\xi-\xi_{p}\right)^{2}\right]\right. \\
& \left.\times \frac{(\xi+q)^{2}+r\left(\xi^{2}+4\right)+q^{2}+2}{\xi^{2}+4} d \xi\right\}^{2} .
\end{aligned}
$$

As in the case of Eq. (25), the integrals in Eq. (36) cannot be evaluated analytically. By applying the same approximation introduced in Sec. III A, however, the probe-laser line shape may be, again, represented by the box function in Eq. (26) and this procedure results in a signal intensity

$$
I=\frac{|S|^{2}\left|\mathcal{E}^{p}\right|^{2}\left|\mathcal{E}^{g}\right|^{4}|\langle i|\mu| e\rangle|^{4}}{(2 \pi)^{15} \Gamma_{e i}^{2}} \frac{L^{2} \omega_{Q B}^{2}}{8 \pi c} \frac{1}{\Delta_{p}^{2}} \mathcal{I}\left(\xi_{p}\right),
$$

where

$$
\mathcal{I}\left(\xi_{p}\right)=\left|\int_{\xi_{p}-\sigma}^{\xi_{p}+\sigma} d \xi \int_{-\infty}^{\infty} d \eta\left[\frac{(q+\eta)^{2}}{1+\eta^{2}}+r\right] \frac{1}{(\xi-\eta+i)}\right|^{2},
$$

and from which, as shown in Eqs. (C9) and (C10) of Appendix $\mathrm{C}$, one can write for the signal line profile

$$
\begin{aligned}
\mathcal{I}\left(\xi_{p}\right)= & \left(q^{2}-1\right) \operatorname{arctanh} \frac{2 \sigma \xi_{p}}{\sigma^{2}+\xi_{p}^{2}+4} \\
& \left.+2 q \arctan \frac{4 \sigma}{\sigma^{2}-\xi_{p}^{2}-4}\right]^{2} \\
& +\left[\left(q^{2}-1\right) \arctan \frac{4 \sigma}{\sigma^{2}-\xi_{p}^{2}-4}\right. \\
& \left.-2 q \operatorname{arctanh} \frac{2 \sigma \xi_{p}}{\sigma^{2}+\xi_{p}^{2}+4}-2(1+r) \sigma\right]^{2} .
\end{aligned}
$$

\section{Discussion}

In Fig. 6, the signal line profiles for a Lorentzian laser line shape, Eq. (34), and for a Gaussian laser line shape, Eq. (38), are plotted in units of $\Gamma / 2$ for three different values of the Fano parameter $q$ and $\sigma$ along with the line profiles obtained for the same values of $q$ but in the case of a $\delta$-function probe laser line shape, where the ratio of the probe laser bandwidth to the Fano states decay rate, $\sigma \rightarrow 0$. Since a normalization extending over all detunings is not possible because the expression for $|\langle e|\mu| \eta)|^{2}$ in Eq. (14) holds only near resonance and is nonintegrable on a large energy scale, 


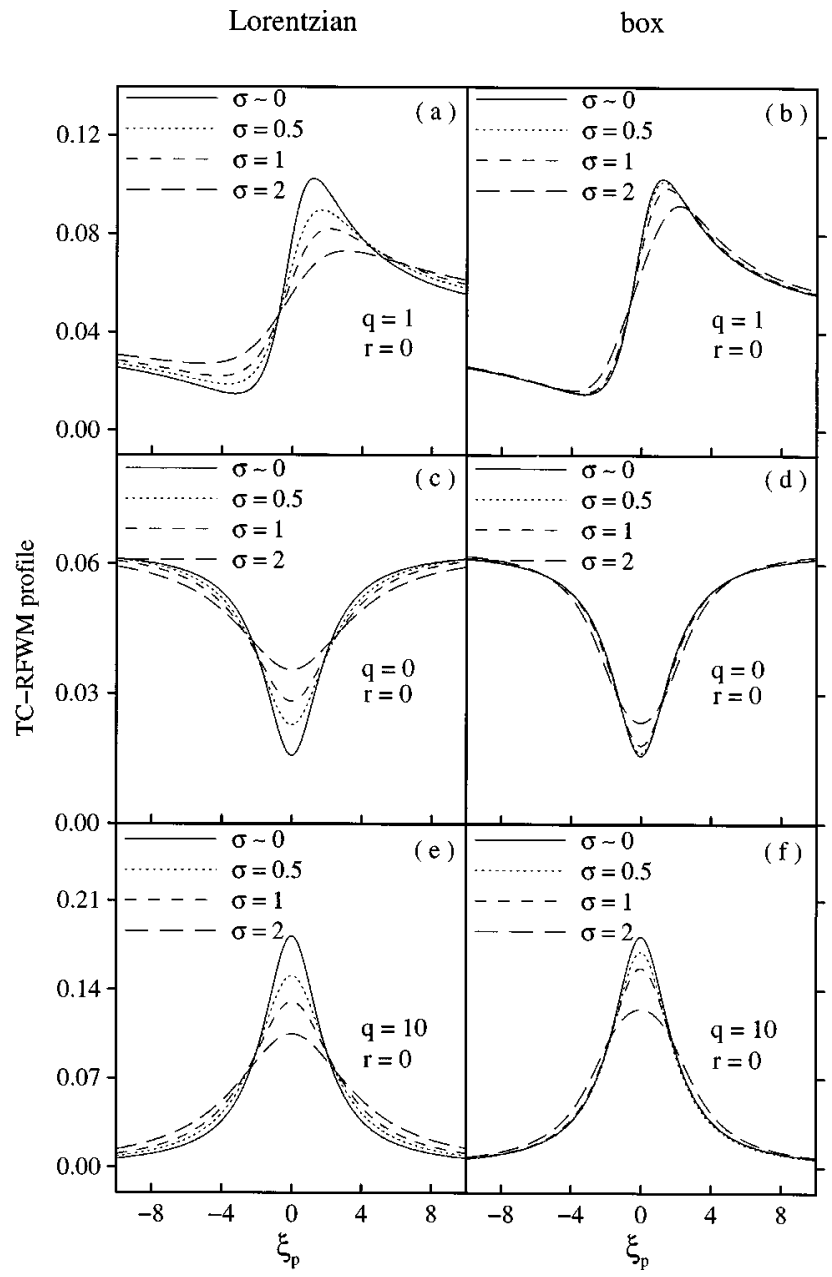

FIG. 6. (a, c, e) Line profiles given by Eq. (34) and (b, d, f) line profiles given by Eq. (38) for different values of $\sigma$ and $q$, with $r=0$. (a, b) $q=1$, (c, d) $q=0,(\mathrm{e}, \mathrm{f}) q=10$.

all of the profiles in Fig. 6 and those in all the other figures pertaining to this section are normalized by the area under the curves calculated on an abscissa interval of -10 to 10 to allow for comparisons. ${ }^{24}$ As is the case for discrete states, the signal line profile obtained by modeling the probe laser with a Lorentzian line shape is more sensitive to the laser bandwidth than that obtained by modeling the probe laser with a box function. In Fig. 6, we observe that for $q=0$ [Figs. 6(c) and 6(d)] and for large values of $q$ [Figs. 6(e) and 6(f)] the variation of $\sigma$ only influences the width of the profiles while, for intermediate values of $q$ [Figs. 6(a) and 6(b)], different values of $\sigma$ result in significant alterations of the profile asymmetries so that, overall, neglecting the laser bandwidth may lead to substantial errors in the estimation of the profile fitting parameters $\Gamma, q$, and $r$.

In Fig. 7 the effect of changing $\sigma$ is shown to have no significant consequence on the wings of the line profile, which are mainly affected by $r$. This can be understood by considering that, on resonance, increasing $\sigma$ corresponds to coherently exciting more Fano states while, far from resonance, the line profile is due to the entire set of Fano states which gives rise, at any value of $\xi_{p}$, to susceptibility terms with nonvanishing dipoles of the form $|\langle e|\mu| \eta)|^{2}$. In other words, the off-resonance structure of the line profile reflects

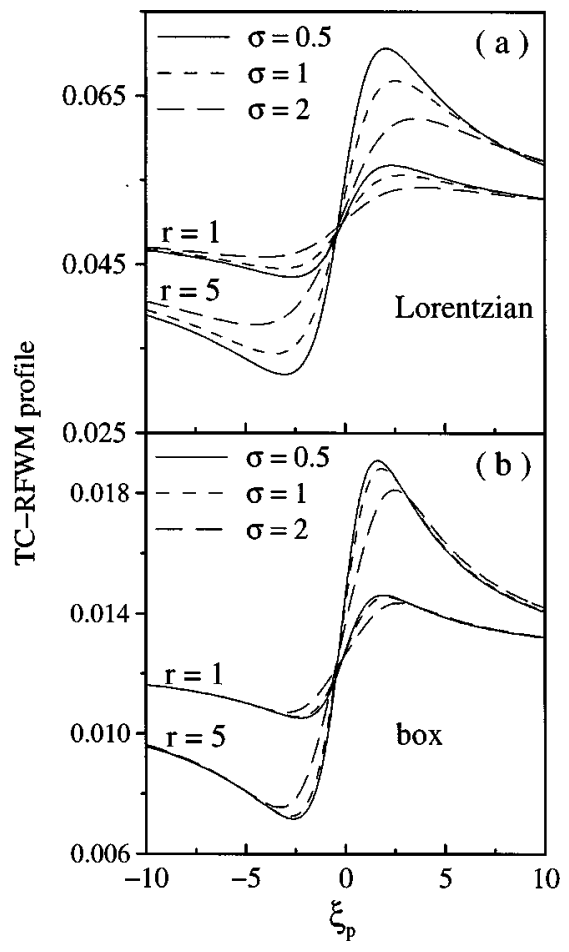

FIG. 7. (a) Line profiles given by Eq. (34) and (b) line profiles given by Eq. (38) for different values of $\sigma$ and $r$.

directly the nonlinear nature of the four-wave mixing process. In Fig. 8 the line profile obtained by approximating the laser line shape with a box function is compared, for the example case of $q=1$, to the line profiles obtained by evaluating numerically the integrals in Eq. (36). As in the case of discrete states, the box-function approximation to the Gaussian line shape is found to be reliable up to $\sigma \approx 1$, and this observation holds for different values of $q$.

\section{CONCLUSIONS}

A theoretical analysis of the effect of the laser line shape and bandwidth on the signal line profile observed by probing either discrete or quasibound state by means of TC-RFWM has been developed. In Sec. II, the third-order polarization responsible for the generation of the detected signal is derived through an $a b$ initio frequency-domain calculation based on a careful application of the phase-matching conditions and the RWA. In Sec. III, the general expressions for the signal profile given in Sec. II are explicitly evaluated by using Lorentzian and Gaussian functions to model the probe laser line shape. The main results of this paper are the theoretical line profiles in Eqs. (22), (26), (34), and (38) which represent analytic functions that can be used directly as fitting functions for the observed TC-RFWM signal. While Eqs. (22) and (34), obtained for the case of Lorentzian probe laser line shape, are exact, Eqs. (26) and (38) are approximations obtained by modeling the Gaussian probe laser line shape as a box function. Such an approximation is shown to be reliable for ratios between the probe laser bandwidth and the width of the probed resonant feature that range from $\sim 0$ to $\sim 1$. 


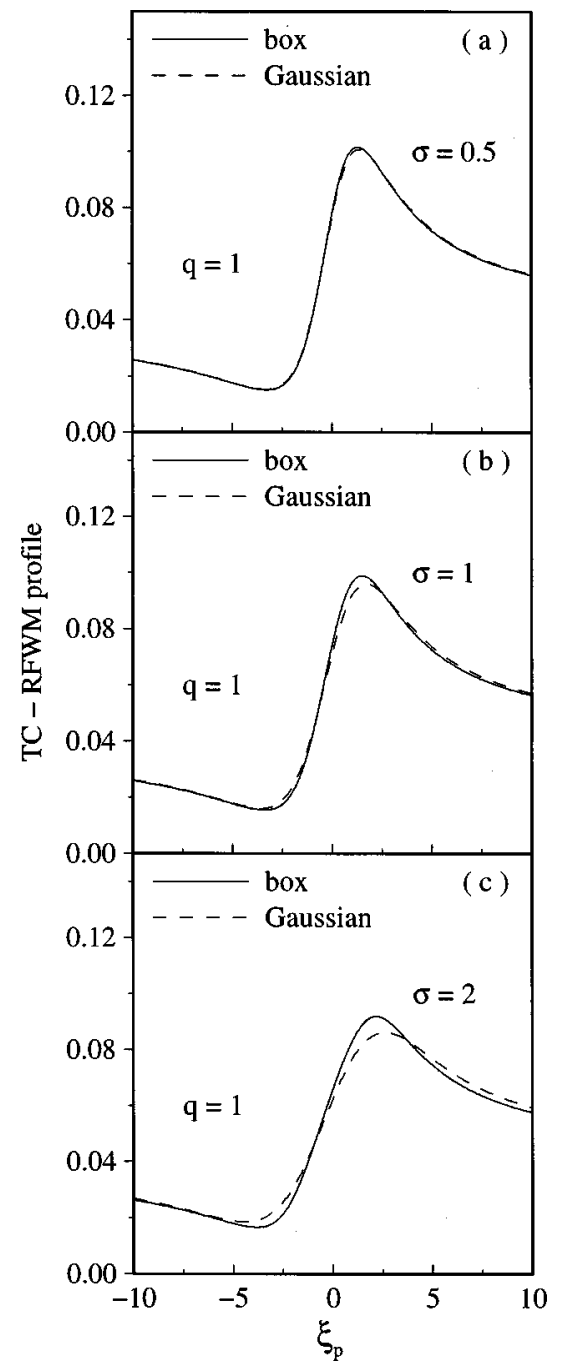

FIG. 8. Comparison of the line profile given by Eq. (38) (solid line) and the line profile given by Eq. (36) for different values of $\sigma$; (a) $\sigma=0.5$, (b) $\sigma$ $=1$, (c) $\sigma=2$.

The line profiles resulting from a Lorentzian probe laser line shape are shown to behave differently from the line profiles obtained by modeling the probe laser line shape as a box function. This implies that the knowledge of both the bandwidth and the line shape of the probe laser is necessary in order to precisely extract from fits to measured signal line profiles quantities of physical interest. This information is particularly important for the case of quasibound states where differences in the probe laser line shape and bandwidth affect not only the width of the resonant feature, but also its asymmetry and off-resonance pedestal.

\section{ACKNOWLEDGMENT}

This work was supported by the National Science Foundation, Grant No. PHY-9623569.

\section{APPENDIX A: DERIVATION OF THE SIGNAL INTENSITY FROM THE EXPRESSION FOR THE POLARIZATION}

\section{Signal intensity for the case of $\delta$-function laser line shapes}

Let us assume that the input laser beams have $\delta$-function line shapes. The induced polarization oscillating at frequency $\omega_{p}$ is, in this case, obtained by replacing, in Eq. (7), $g(\omega$ $\left.\pm \omega_{g}, \Delta_{g}\right)$ and $p\left(\omega \pm \omega_{p}, \Delta_{p}\right)$ with $\delta\left(\omega \pm \omega_{g}\right)$ and $\delta(\omega$ $\left.\pm \omega_{p}\right)$, respectively, and evaluating the integrals over the frequencies. The result is

$$
\begin{aligned}
P^{(3)}(\mathbf{r}, t)= & {\left[P_{\delta} \exp \left(-i \omega_{p} t+i \mathbf{k}_{s} \cdot \mathbf{r}\right)+P_{\delta} \exp \left(-i \omega_{p} t\right.\right.} \\
& \left.\left.+i \mathbf{k}_{s}^{\prime} \cdot \mathbf{r}\right)\right]+ \text { c.c. },
\end{aligned}
$$

where

$$
\begin{aligned}
& P_{\delta}=\frac{\chi^{(3)}\left(\omega_{p}, \omega_{g},-\omega_{g}\right) \mathcal{E}^{p}\left|\mathcal{E}^{g}\right|^{2}}{(2 \pi)^{3}}, \\
& \mathbf{k}_{s}=\frac{\omega_{p}}{c} \hat{\mathbf{k}}_{p}+\frac{\omega_{g}}{c}\left(\hat{\mathbf{k}}_{g 1}-\hat{\mathbf{k}}_{g 2}\right), \\
& \mathbf{k}_{s}^{\prime}=\frac{\omega_{p}}{c} \hat{\mathbf{k}}_{p}-\frac{\omega_{g}}{c}\left(\hat{\mathbf{k}}_{g 1}-\hat{\mathbf{k}}_{g 2}\right) .
\end{aligned}
$$

Note, however, that both $\left|\mathbf{k}_{s}\right|$ and $\left|\mathbf{k}_{s}^{\prime}\right|$ should be equal to $\omega_{p} / c$, which is possible only when $\hat{\mathbf{k}}_{g 1}=\hat{\mathbf{k}}_{g 2}$ and, therefore, $\mathbf{k}_{s}=\mathbf{k}_{s}^{\prime}=\left(\omega_{p} / c\right) \hat{\mathbf{k}}_{p}$. If $\hat{\mathbf{k}}_{g 1} \neq \hat{\mathbf{k}}_{g 2}$, the direction of the polarization wave vector is uniquely determined by the Bragg scattering condition, as shown in Appendix A of Ref. 24. The polarization $P^{(3)}(\mathbf{r}, t)$ can, therefore, be expressed as a plane wave

$$
P^{(3)}(\mathbf{r}, t)=P_{\delta} \exp \left(-i \omega_{p} t+i \mathbf{k}_{s} \cdot \mathbf{r}\right)+\text { c.c., }
$$

where $\mathbf{k}_{s}=\left(\omega_{p} / c\right) \hat{\mathbf{k}}_{p}+\left(\omega_{g} / c\right)\left(\hat{\mathbf{k}}_{g 1}-\hat{\mathbf{k}}_{g 2}\right)$. By applying the RWA in the same manner as in Sec. II A 3, the expression of the susceptibility $\chi^{(3)}\left(\omega_{p}, \omega_{g},-\omega_{g}\right)$ for the excitation scheme depicted in Fig. 1(a) becomes ${ }^{23}$

$$
\begin{aligned}
\chi^{(3)}\left(\omega_{p}, \omega_{g},-\omega_{g}\right) \\
=S|\langle i|\mu| e\rangle|^{2}|\langle e|\mu| f\rangle|^{2} \frac{1}{\omega_{p}-\omega_{f e}+i \Gamma_{f e}} \\
\quad \times\left(\frac{1}{\left(\omega_{g}-\omega_{e i}+i \Gamma_{e i}\right)}+\frac{1}{\left(-\omega_{g}+\omega_{e i}+i \Gamma_{e i}\right)}\right) .
\end{aligned}
$$

The polarization in Eq. (A3) may be substituted in Eq. (1) and treated as a source for the optical field of the signal beam. A solution of Eq. (1) can be obtained in the form of plane wave as

$$
E(\mathbf{r}, t)=E(z, t) \exp \left(-i \omega_{p} t+i \tilde{\mathbf{k}}_{s} \cdot \mathbf{r}\right)+\text { c.c. },
$$

where

$$
\begin{aligned}
& z \equiv \frac{\widetilde{\mathbf{k}}_{s} \cdot \mathbf{r}}{\left|\widetilde{\mathbf{k}}_{s}\right|}, \\
& \left|\widetilde{\mathbf{k}}_{s}\right|=\frac{\omega_{p}}{c} \simeq \frac{\omega_{f e}}{c},
\end{aligned}
$$

and $E(z, t)$ is a temporally and spatially slowly-varying function. By substituting Eq. (A5) into Eq. (1), the following differential equation is obtained:

$$
i \frac{\omega_{f e}}{c} \frac{\partial E}{\partial z}=-2 \pi \frac{\omega_{f e}^{2}}{c^{2}} P_{\delta} \exp \left[i\left(\mathbf{k}_{s}-\widetilde{\mathbf{k}}_{s}\right) \cdot \mathbf{r}\right] .
$$


Here the slowly varying envelope approximations,

$$
\begin{aligned}
& \left|\frac{\partial^{2} E}{\partial t^{2}}\right| \ll\left|\omega_{p} E\right|^{2}, \\
& \left|\frac{\partial^{2} E}{\partial z^{2}}\right| \ll \mid \widetilde{\mathbf{k}}_{s} E^{2},
\end{aligned}
$$

have been used. By integrating Eq. (A7) over the spatial extent of the material medium along the direction identified by $\widetilde{\mathbf{k}}_{s}$, an explicit expression for $E$ is obtained, thereby allowing us to write the intensity $I$ of the detected signal in the case of $\delta$-function input laser beams as

$$
I \equiv \frac{c}{8 \pi}|E|^{2}=\frac{\pi}{2 c} L^{2}\left|\omega_{f e} P_{\delta}\right|^{2}\left(\frac{\sin \vartheta}{\vartheta}\right)^{2}
$$

In Eq. (A9) $L$ is the path length through the medium along the direction $\widetilde{\mathbf{k}}_{s}$ and $\vartheta \equiv\left|\mathbf{k}_{s}-\widetilde{\mathbf{k}}_{s}\right| L$. Since $(\sin \vartheta / \vartheta)$ peaks at zero, optimal phase matching is achieved for $\mathbf{k}_{s}=\widetilde{\mathbf{k}}_{s}$. These conditions along with Eq. (A6b) uniquely determine the propagation direction of the plane wave in Eq. (A5).

The angles $\theta$ between this propagation direction and the probe beam can be obtained by following the procedure introduced in Appendix A of Ref. 24. This results in

$$
\theta=2 \arcsin \left(\frac{\omega_{g}}{\omega_{p}} \sin \frac{\theta_{g}}{2}\right)
$$

where $\theta_{g}$ is the angle between the grating beams.

\section{Signal intensity for the case of finite-bandwidth laser line shapes}

Let us assume that the input laser beams have finitebandwidth line shapes represented by the functions $g(\omega$ $\left.\pm \omega_{g}, \Delta_{g}\right)$ and $p\left(\omega \pm \omega_{p}, \Delta_{p}\right)$. The resulting induced polarization oscillating at frequency $\omega_{p}$ and characterized by the wave vector $\mathbf{k}_{s}$ is given by Eq. (10). Thus, a solution $E(\mathbf{r}, t)$ of Eq. (1) may be found in the form of

$$
E(\mathbf{r}, t)=E(z, t) \exp \left(i \tilde{\mathbf{k}}_{s} \cdot \mathbf{r}\right)+\text { c.c. }
$$

By substituting Eqs. (10) and (A11) into Eq. (1) and Fouriertransforming both sides of this equation, the linear differential equation

$$
\begin{aligned}
i \frac{\omega_{f e}}{c} \frac{\partial E(z, \omega)}{\partial z}= & -\frac{2 \pi \omega^{2}}{c^{2}} \frac{p\left(\omega-\omega_{p}, \Delta_{p}\right)}{\left(\omega-\omega_{f e}+i \Gamma_{f e}\right)} \\
& \times P \exp \left[i\left(\mathbf{k}_{s}-\widetilde{\mathbf{k}}_{s}\right) \cdot \mathbf{r}\right]+\frac{\omega_{f e}^{2}-\omega^{2}}{c^{2}} E(z, \omega),
\end{aligned}
$$

is obtained. Here Eq. (11) has been used and the approximations in Eqs. (A8) have been adopted for $E(\mathbf{r}, t)$. For $\omega$ $\simeq \omega_{p} \simeq \omega_{f e}$, Eq. (A12) may be rewritten as

$$
\begin{aligned}
i \frac{\omega_{f e}}{c} \frac{\partial E(z, \omega)}{\partial z}= & -\frac{2 \pi \omega_{f e}^{2}}{c^{2}} \frac{p\left(\omega-\omega_{p}, \Delta_{p}\right)}{\left(\omega-\omega_{f e}+i \Gamma_{f e}\right)} \\
& \times P \exp \left[i\left(\mathbf{k}_{s}-\widetilde{\mathbf{k}}_{s}\right) \cdot \mathbf{r}\right],
\end{aligned}
$$

and integrated over $z$ for the interval $(0, L)$ to give

$$
E(L, \omega)=\frac{2 \pi i \omega_{f e}}{c} \frac{p\left(\omega-\omega_{p}, \Delta_{p}\right)}{\left(\omega-\omega_{f e}+i \Gamma_{f e}\right)} P L \exp (i \vartheta) \frac{\sin \vartheta}{\vartheta}
$$

The phase-matched $(\vartheta=0)$ detected signal $I$, which results from the coherent superposition of the Fourier components of the output optical field, is given by

$$
\begin{aligned}
I & \equiv \frac{c}{8 \pi}\left|\int_{-\infty}^{\infty} E(L, \omega) d \omega\right|^{2} \\
& =\frac{|P|^{2} L^{2} \omega_{f e}^{2}}{8 \pi c}\left|\int_{-\infty}^{\infty} d \omega \frac{p\left(\omega-\omega_{p}, \Delta_{p}\right)}{\left(\omega-\omega_{f e}+i \Gamma_{f e}\right)}\right|^{2},
\end{aligned}
$$

which is the expression given in Eq. (12). Similarly, to obtain Eq. (17), it suffices to replace $\omega_{f e}$ with $\omega_{Q B}$ in Eq. (A15) and make use of the expression of the susceptibility given in Eq. (15).

\section{APPENDIX B: EVALUATION OF THE GRATING- LASER CONTRIBUTION TO THE SIGNAL LINE PROFILE}

The integral over $\omega_{1}$ and $\omega_{2}$ in Eq. (10) may be written as

$$
\begin{aligned}
j(t)= & \int_{-\infty}^{\infty} e^{-i \omega t} g\left(\omega+\omega_{g}, \Delta_{g}\right) d \omega \int_{-\infty}^{\infty} e^{-i \omega t} \\
& \times \frac{g\left(\omega-\omega_{g}, \Delta_{g}\right)}{\omega-\omega_{e i}+i \Gamma_{e i}} d \omega+\int_{-\infty}^{\infty} e^{-i \omega t} g\left(\omega-\omega_{g}, \Delta_{g}\right) d \omega \\
& \times \int_{-\infty}^{\infty} e^{-i \omega t} \frac{g\left(\omega+\omega_{g}, \Delta_{g}\right)}{-\omega+\omega_{e i}+i \Gamma_{e i}} d \omega,
\end{aligned}
$$

where the numerical subscripts of the frequencies have been dropped for simplicity. Thus, Eq. (10) may be rewritten as

$$
P^{(3)}(\mathbf{r}, t)=P \exp \left(i \mathbf{k}_{s} \cdot \mathbf{r}\right) j(t) \int_{-\infty}^{\infty} H\left(\omega_{3}\right) e^{-i \omega_{3} t} d \omega_{3},
$$

where

$$
H\left(\omega_{3}\right)=\frac{p\left(\omega_{3}-\omega_{p}, \Delta_{p}\right)}{\omega_{3}-\omega_{f e}+i \Gamma_{f e}},
$$

and the time Fourier transform of $P^{(3)}(\mathbf{r}, t)$ is

$$
\begin{aligned}
& P^{(3)}(\mathbf{r}, \omega) \\
& \quad=P \exp \left(i \mathbf{k}_{s} \cdot \mathbf{r}\right) \int_{-\infty}^{\infty}\left[e^{i \omega t} j(t) d \omega \int_{-\infty}^{\infty} H\left(\omega_{3}\right) e^{-i \omega_{3} t}\right] d \omega_{3} \\
& \quad=P \exp \left(i \mathbf{k}_{s} \cdot \mathbf{r}\right) \int_{-\infty}^{\infty} H\left(\omega_{3}\right) d \omega_{3} \int_{-\infty}^{\infty} e^{i \omega t} e^{-i \omega_{3} t} j(t) d \omega \\
& \quad=P \exp \left(i \mathbf{k}_{s} \cdot \mathbf{r}\right) \int_{-\infty}^{\infty} H\left(\omega_{3}\right) J\left(\omega-\omega_{3}\right) d \omega_{3} .
\end{aligned}
$$

In Eq. (B3), $J(\omega)$ is the Fourier transform of $j(t)$. According to Eq. (B1) and owing to the convolution theorem for Fourier transforms, we obtain 


$$
\begin{aligned}
J(\omega)= & \int_{-\infty}^{\infty} g\left(\Omega+\omega_{g}, \Delta_{g}\right) \frac{g\left(\omega-\Omega-\omega_{g}, \Delta_{g}\right)}{\omega-\Omega-\omega_{e i}+i \Gamma_{e i}} d \Omega \\
& +\int_{-\infty}^{\infty} g\left(\Omega-\omega_{g}, \Delta_{g}\right) \frac{g\left(\omega-\Omega+\omega_{g}, \Delta_{g}\right)}{-\omega-\Omega+\omega_{e i}+i \Gamma_{e i}} d \Omega .
\end{aligned}
$$

The first term in Eq. (B4) is the overlap integral between $g\left(\Omega+\omega_{g}, \Delta_{g}\right)$, which has a maximum at $\Omega=-\omega_{g}$, and $g\left(\omega-\Omega-\omega_{g}, \Delta_{g}\right) /\left(\omega-\Omega-\omega_{e i}+i \Gamma_{e i}\right)$, which, given that $\omega_{g} \simeq \omega_{e i}$, has a maximum at $\Omega=\omega-\omega_{g}$. Assuming that the spread of all functions around their maxima is much smaller than $2 \omega_{g}$ and knowing that the horizontal centroids of two functions add under convolution, maximum overlap is obtained when $\omega-\omega_{g}=-\omega_{g}$, i.e., when $\omega=0$. Owing to the same arguments, the second term also exhibits a maximum for $\omega=0$. Hence, the overall $J(\omega)$ has a maximum at $\omega$ $=0$. In light of these facts, Eq. (B2) implies that the timedomain polarization $P^{(3)}(\mathbf{r}, t)$ is the product of $j(t)$, a slowly-varying function, times the Fourier antitransform of $H(\omega)$, which oscillates at a rapidly-varying optical frequency, namely, $\omega \simeq \omega_{p} \simeq \omega_{f e}$. Thus, $j(t)$ results only in a slow amplitude-modulation having negligible effect on the optical spectrum, which allows us to use the approximation $J\left(\omega-\omega_{3}\right) \simeq J_{0} \delta\left(\omega-\omega_{3}\right)$, where $J_{0}$ is a constant. This approximation is equivalent to modeling the grating beam line shape as a $\delta$ function. Since $\omega_{g} \simeq \omega_{e i}$, Eq. (B4) can, indeed, be rewritten as

$$
\begin{aligned}
J(\omega)= & \int_{-\infty}^{\infty} g\left(x, \Delta_{g}\right) \frac{g\left(\omega-x, \Delta_{g}\right)}{\omega-x+i \Gamma_{e i}} d x \\
& +\int_{-\infty}^{\infty} g\left(y, \Delta_{g}\right) \frac{g\left(\omega-y, \Delta_{g}\right)}{-\omega-y+i \Gamma_{e i}} d y,
\end{aligned}
$$

where $x=\Omega+\omega_{g}$ and $y=\Omega-\omega_{g}$. Equation (B5) can be written as

$$
\begin{aligned}
J(\omega)= & \int_{-\infty}^{\infty} g\left(x, \Delta_{g}\right) g\left(\omega-x, \Delta_{g}\right) \\
& \times\left(\frac{1}{\omega-x+i \Gamma_{e i}}+\frac{1}{-\omega-x+i \Gamma_{e i}}\right) d x \\
= & \int_{-\infty}^{\infty} g\left(x, \Delta_{g}\right) g\left(\omega-x, \Delta_{g}\right) \frac{x-i \Gamma_{e i}}{\omega^{2}-\left(x-i \Gamma_{e i}\right)^{2}} d x,
\end{aligned}
$$

and, by approximating $g\left(x, \Delta_{g}\right)$ and $g\left(\omega-x, \Delta_{g}\right)$ with $\delta(x)$ and $\delta(\omega-x)$, respectively, we obtain

$$
J(\omega) \simeq \int_{-\infty}^{\infty} \delta(x) \delta(\omega-x) \frac{x-i \Gamma_{e i}}{\omega^{2}-\left(x-i \Gamma_{e i}\right)^{2}} d x=\frac{\delta(\omega)}{\Gamma_{e i}} .
$$

According to Eq. (B6), it is also seen that $J_{0}=1 / \Gamma_{e i}$, which allows us to rewrite Eq. (B3) as

$$
P^{(3)}(\mathbf{r}, \omega)=\frac{P}{\Gamma_{e i}} \exp \left(i \mathbf{k}_{s} \cdot \mathbf{r}\right) \int_{-\infty}^{\infty} H\left(\omega_{3}\right) \delta\left(\omega-\omega_{3}\right) d \omega_{3}
$$

By evaluating the integral in Eq. (B7), Eq. (11) is obtained. The same arguments can also be used to derive Eq. (16).

\section{APPENDIX C: EVALUATION OF THE SIGNAL LINE-PROFILE INTEGRALS}

The evaluation of the integral in Eq. (21) may be performed by means of contour integration in the complex plane. A complex variable $z$ is introduced such that $\zeta$ $=\operatorname{Re}(z)$, the integrand of Eq. (21) is evaluated in $z$, and three poles of first order are found, $z_{\text {pole }}=-2 i, \zeta_{p} \pm i \beta$. Choosing the semicircle path $\mathcal{C}$ shown in Fig. 9 and applying the residue theorem yields

$$
\begin{aligned}
\mathcal{I}\left(\zeta_{p}\right)= & 2 \pi i \operatorname{Res}\left(\zeta_{p}+i \beta\right)+\lim _{\mathcal{R} \rightarrow \infty} \\
& \times\left.\int_{0}^{\pi} \frac{i \mathcal{R} e^{i \theta} d \theta}{\mathcal{R}^{3}\left[\left(e^{i \theta}-\zeta_{p} / \mathcal{R}\right)^{2}+\beta^{2} / \mathcal{R}^{2}\right]\left(e^{i \theta}+2 i / \mathcal{R}\right)}\right|^{2} \\
= & \left|2 \pi i \operatorname{Res}\left(\zeta_{p}+i \beta\right)\right|^{2}=\frac{\pi^{2}}{\beta^{2}}\left|\frac{1}{\zeta_{p}+i(\beta+2)}\right|^{2}
\end{aligned}
$$

where $\operatorname{Res}\left(\zeta_{p}+i \beta\right)$ is the residue of the integrand in $z=\zeta_{p}$ $+i \beta$ and $\mathcal{R}$ is the radius of $\mathcal{C}$. By evaluating the absolute square in Eq. (C1), Eq. (22) is obtained.

Complex-plane contour integration can also be used to evaluate the integral over $\eta$ in Eq. (33) and, as shown in Eq. (B1) of Ref. 24, this results in

$$
\begin{aligned}
\mathcal{I}\left(\xi_{p}\right)= & \mid \int_{-\infty}^{\infty} d \xi \frac{1}{\left(\xi-\xi_{p}\right)^{2}+\sigma^{2}} \\
& \times\left.\frac{\left(q^{2}+2 r+1\right)-i[2 q+(1+r) \xi]}{\xi+2 i}\right|^{2} .
\end{aligned}
$$

At this point, the integration over $\xi$ can be performed, again, via complex-plane contour integration by introducing the complex variable $z$ such that $\operatorname{Re}(z)=\xi$ and exploiting the same path $\mathcal{C}$ shown in Fig. 9. Again, three poles are found; $z_{\text {pole }}=-2 i, \xi_{p} \pm i \sigma$ and, using the residue theorem, the following result is obtained:

$$
\begin{aligned}
\mathcal{I}\left(\xi_{p}\right)= & \mid 2 \pi i \operatorname{Res}\left(\xi_{p}+i \sigma\right)+\lim _{\mathcal{R} \rightarrow \infty} \int_{0}^{\pi} i e^{i \theta} d \theta \\
& \times\left.\frac{\left(q^{2}+2 r+1\right) / \mathcal{R}-i\left[2 q / \mathcal{R}+(1+r) e^{i \theta}\right]}{\mathcal{R}\left[\left(e^{i \theta}-\xi_{p} / \mathcal{R}\right)^{2}+\sigma^{2} / \mathcal{R}^{2}\right]\left(e^{i \theta}+2 i / \mathcal{R}\right)}\right|^{2} \\
= & \left|2 \pi i \operatorname{Res}\left(\xi_{p}+i \sigma\right)\right|^{2} \\
= & \left|\frac{\pi}{\sigma} \frac{\left(q^{2}+2 r+1\right)-i\left[2 q+(1+r)\left(\xi_{p}+i \sigma\right)\right]}{\xi_{p}+i \sigma+2 i}\right|^{2},
\end{aligned}
$$




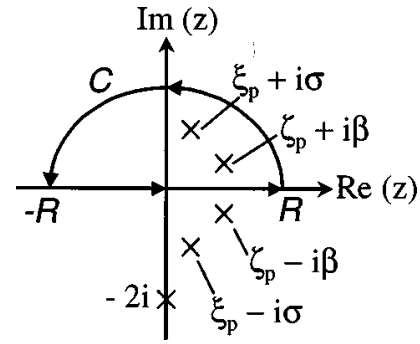

FIG. 9. Integration path in the complex plane used to evaluate Eq. (21). The same path can be used to evaluate Eq. (33), by replacing $\zeta_{p} \pm i \beta$ with $\xi_{p}$ $\pm i \sigma$

which, after the evaluation of the squared modulus, gives the expression in Eq. (34).

The evaluation of Eq. (28) is performed by observing that

$$
\begin{aligned}
\mathcal{I}\left(\zeta_{p}\right)= & \left|\int_{\zeta_{p}-\beta}^{\zeta_{p}+\beta} \frac{1}{\zeta+2 i} d \zeta\right|^{2}=\left(\int_{\zeta_{p}-\beta}^{\zeta_{p}+\beta} \frac{\zeta}{\zeta^{2}+4} d \zeta\right)^{2} \\
& +4\left(\int_{\zeta_{p}-\beta}^{\zeta_{p}+\beta} \frac{1}{\zeta^{2}+4} d \zeta\right)^{2},
\end{aligned}
$$

where the antiderivatives of the two integrands in parentheses are $\ln \sqrt{\zeta^{2}+4}$ and $(1 / 2) \arctan (\zeta / 2)$, respectively. The result in Eq. (29) is then obtained by using the addition rule for the inverse tangent,

$$
\arctan x-\arctan y=\arctan \frac{x-y}{1+x y}, \quad \forall x, y,
$$

along with the identity

$$
\frac{1}{2} \ln \frac{1+x}{1-x}=\operatorname{arctanh} x, \quad \forall x \text {. }
$$

The integral in Eq. (38) may, finally, be rewritten as

$$
\mathcal{I}\left(\xi_{p}\right)=\left|\int_{\xi_{p}-\sigma}^{\xi_{p}+\sigma} d \xi \frac{\left(q^{2}+2 r+1\right)-i[2 q+(1+r) \xi]}{\xi+2 i}\right|^{2}
$$

by performing the integration over $\eta$ as done above to obtain Eq. (C2). The integration over $\xi$ in Eq. (C7) may be performed by introducing once again the complex variable $z$ such that $\xi=\operatorname{Re}(z)$, evaluating the integrand in $z$, and calculating the integral in the complex plane. The integral may, then, be rewritten as

$$
\begin{aligned}
\mathcal{I}\left(\xi_{p}\right)= & \left(q^{2}+2 r+1-2 i q\right) \int_{\xi_{p}-\sigma}^{\xi_{p}+\sigma} \frac{d z}{z+2 i} \\
& -\left.i(1+r) \int_{\xi_{p}-\sigma}^{\xi_{p}+\sigma}\left(1-\frac{2 i}{z+2 i}\right) d z\right|^{2} \\
= & \left|(q-i)^{2} \int_{\xi_{p}-\sigma}^{\xi_{p}+\sigma} \frac{d z}{z+2 i}-i(1+r) \int_{\xi_{p}-\sigma}^{\xi_{p}+\sigma} d z\right|^{2} .
\end{aligned}
$$

The integrand in Eq. (C8) has only one pole at $\xi=-2 i$ and it is analytic in a connected region including the real-axis path $\left[\left(\xi_{p}-\sigma\right) \rightarrow\left(\xi_{p}+\sigma\right)\right]$ so that, owing to the Cauchy theorem,

$$
\begin{aligned}
\mathcal{I}\left(\xi_{p}\right)= & \mid(q-i)^{2}\left[\ln \left(\xi_{p}+\sigma+2 i\right)-\ln \left(\xi_{p}-\sigma+2 i\right)\right] \\
& -\left.2 i(1+r) \sigma\right|^{2} \\
= & \mid(q-i)^{2}\left[\ln \frac{\sqrt{\left(\xi_{p}+\sigma\right)^{2}+4}}{\sqrt{\left(\xi_{p}-\sigma\right)^{2}+4}}\right. \\
& \left.+i\left(\arctan \frac{2}{\xi_{p}+\sigma}-\arctan \frac{2}{\xi_{p}-\sigma}\right)\right] \\
& -\left.2 i(1+r) \sigma^{2}\right|^{2} .
\end{aligned}
$$

By using Eqs. (C5) and (C6), Eq. (C9) may be written as

$$
\begin{aligned}
\mathcal{I}\left(\xi_{p}\right)= & \mid(q-i)^{2}\left[\operatorname{arctanh} \frac{2 \sigma \xi_{p}}{\sigma^{2}+\xi_{p}^{2}+4}\right. \\
& \left.+i \arctan \frac{4 \sigma}{\sigma^{2}-\xi_{p}^{2}-4}\right]-\left.2 i(1+r) \sigma^{2}\right|^{2} .
\end{aligned}
$$

By explicitly calculating the squared modulus at the righthand side of Eq. (C10), the result in Eq. (38) is obtained.

${ }^{1}$ R. L. Farrow and D. J. Rakestraw, Science 257, 1894 (1992).

${ }^{2}$ D. S. Green, T. G. Owano, S. Williams, D. G. Goodwin, R. N. Zare, and C. H. Kruger, Science 259, 1726 (1993).

${ }^{3}$ P. H. Vaccaro, in Molecular Dynamics and Spectroscopy by Stimulated Emission Pumping, edited by H. L. Dai and R. W. Field, Advances in Physical Chemistry Series, edited by C.-Y. Ng (World Scientific, Singapore, 1995), Vol. 4, p. 1.

${ }^{4}$ J. T. Fourkas and M. D. Fayer, Acc. Chem. Res. 25, 227 (1992).

${ }^{5}$ G. Hall and J. Whittaker, J. Chem. Soc., Faraday Trans. 90, 1 (1994).

${ }^{6}$ R. W. Boyd, Nonlinear Optics (Academic, Boston, 1992); P. N. Butcher and D. Cotter, The Elements of Nonlinear Optics (Cambridge University Press, Cambridge, 1990); Y. R. Shen, The Principles of Nonlinear Optics (Wiley, New York, 1984).

${ }^{7}$ R. L. Farrow, D. J. Rakestraw, and T. Dreier, J. Opt. Soc. Am. B 9, 1770 (1992); T. J. Buthenoff and E. A. Rohlfing, J. Chem. Phys. 97, 1595 (1992); E. F. McCormack, S. T. Pratt, P. M. Dehmer, and J. L. Dehmer, Chem. Phys. Lett. 211, 143 (1993).

${ }^{8}$ E. F. McCormack, P. M. Dehmer, J. L. Dehmer, and S. T. Pratt, J. Chem. Phys. 102, 4740 (1995); Aust. J. Phys. 49, 445 (1996).

${ }^{9}$ M. A. Buntine, D. W. Chandler, and C. C. Hayden, J. Chem. Phys. 97, 707 (1992).

${ }^{10}$ T. J. Butenhoff and E. A. Rohlfing, J. Chem. Phys. 98, 5460 (1993); 98, 5469 (1993).

${ }^{11}$ M. D. Wheeler, I. R. Lambert, and M. N. R. Ashfold, Chem. Phys. Lett 229, 285 (1994).

${ }^{12}$ M. N. R. Ashfold, D. W. Chandler, C. C. Hayden, R. I. McKay, and A. J. R. Heck, Chem. Phys. 201, 237 (1995).

${ }^{13}$ M. A. Buntine, D. W. Chandler, and C. C. Hayden, J. Chem. Phys. 102, 2718 (1995).

${ }^{14}$ T. Ebata, A. Okazaki, Y. Inokuchi, and N. Mikami, J. Mol. Struct. 352/ 353, 533 (1995).

${ }^{15}$ W.-C. Hung, M.-L. Huang, Y.-C. Lee, and Y.-P. Lee, J. Chem. Phys. 103, 9941 (1995).

${ }^{16}$ J. Ishii, K. Uehara, and K. Tsukiyama, J. Chem. Phys. 102, 9174 (1995).

${ }^{17}$ J. D. Tobiason, J. R. Dunlop, and E. A. Rohlfing, J. Chem. Phys. 103, 1448 (1995).

${ }^{18}$ S. Williams, J. D. Tobiason, J. R. Dunlop, and E. A. Rohlfing, J. Chem. Phys. 102, 8342 (1995). 
${ }^{19}$ J. Geng, T. Kobayashi, and M. Takami, Chem. Phys. Lett. 266, 290 (1997).

${ }^{20}$ P. P. Radi, H.-M. Frey, B. Mischler, A. P. Tzannis, P. Beaud, and T. Gerber, Chem. Phys. Lett. 265, 271 (1997).

${ }^{21}$ T. Kobayashi, J. Geng, and M. Takami, Chem. Phys. Lett. 284, 195 (1998).

${ }^{22}$ E. F. McCormack, F. Di Teodoro, J. M. Grochocinski, and S. T. Pratt, J. Chem. Phys. 109, 63 (1998).

${ }^{23}$ S. Williams, E. A. Rohlfing, L. A. Rahn, and R. Zare, J. Chem. Phys. 106, 3090 (1997).

${ }^{24}$ F. Di Teodoro and E. F. McCormack, Phys. Rev. A 57, 162 (1998).

${ }^{25}$ A. Dreizler, T. Dreier, and J. Wolfrum, Chem. Phys. Lett. 233, 525 (1995); P. M. Danhey, P. H. Paul, and R. I. Farrow, J. Opt. Soc. Am. B 12, 1564 (1995).

${ }^{26}$ D. E. Govoni, J. A. Bronze, A. Sinha, and F. F. Crim, Chem. Phys. Lett. 216, 525 (1993); B. Hemmerling, R. Bombach, and W. Hubschmid, ibid. 256, 71 (1996).

${ }^{27}$ H. J. Eichler, P. Gunter, and D. W. Pohl, Laser-Induced Dynamic Gratings (Springer, Berlin, 1986).

${ }^{28}$ J. T. Fourkas, R. Trebino, and M. D. Fayer, J. Chem. Phys. 97, 69 (1992); 97, 78 (1992).
${ }^{29}$ T. Muller and P. H. Vaccaro, Chem. Phys. Lett. 266, 575 (1997).

${ }^{30}$ S. Mukamel, Phys. Rev. A 28, 3480 (1983).

${ }^{31}$ P. Zoller, G. Alber, and R. Salvador, Phys. Rev. A 24, 398 (1981).

${ }^{32}$ D. R. Meacher, P. G. R. Smith, P. Ewart, and J. Cooper, Phys. Rev. A 46, 2718 (1992); P. G. R. Smith and P. Ewart, ibid. 54, 2347 (1996).

${ }^{33}$ R. Bratfalean and P. Ewart, Phys. Rev. A 56, 2267 (1997).

${ }^{34}$ Y. Tang and S. A. Reid, J. Chem. Phys. 105, 8481 (1996).

${ }^{35}$ S. Mukamel, Principles of Nonlinear Optical Spectroscopy (Oxford University Press, New York, 1995), see Chap. 4 for the wave equation of the induced field and Chap. 6 for the general expression for the third-order electric susceptibility.

${ }^{36}$ U. Fano, Phys. Rev. 124, 1866 (1961); for a more recent review see P. L. Knight, M. A. Lauder, and B. J. Dalton, Phys. Rep. 190, 1 (1990).

${ }^{37}$ U. Fano and J. W. Cooper, Phys. Rev. A 137, A1364 (1968).

${ }^{38}$ A. Corney, Atomic and Laser Spectroscopy (Clarendon, Oxford, 1977), pp. $253-257$.

${ }^{39}$ S. N. Dobryakov and Ya. S. Lebedev, Sov. Phys. Dokl. 13, 873 (1969).

${ }^{40}$ See, for example, Chap. 3 of R. Bracewell, The Fourier Transform and its Applications (McGraw-Hill, New York, 1965). 